Geophysical and Astrophysical Fluid Dynamics

Vol. 00, No. 00, 00 Month 2014, 1-28

\title{
A laboratory study of global-scale wave interactions in baroclinic flow with topography II: vacillations and low-frequency variability
}

\author{
S. H. RISCH†† and P. L. READ $\dagger^{*}$ \\ $†$ Atmospheric, Oceanic \& Planetary Physics, Department of Physics, University of Oxford, Clarendon \\ Laboratory, Parks Road, Oxford, OX1 3PU, UK \\ †Present affiliation: ESG, Munich, Germany
}

(29 April 2010)

\begin{abstract}
A laboratory investigation is presented with the aim of studying systematically the occurrence and characteristics of low-frequency variability of flows resulting from the interaction of a baroclinic flow with periodic bottom topography. Low-frequency variability within the baroclinic wave regime occurred in two distinct forms in separate regions of parameter space. One corresponded to the transition region between the baroclinic travelling and stationary wave regimes. It involved primarily an interaction between the drifting baroclinic waves and stationary components of the topographically forced wave. The resulting had characteristics similar to amplitude vacillation and had a time-scale of 30-60 annulus revolutions ('days'), which also corresponded with the wave drift period. A new regime of low-frequency amplitude vacillation was discovered in the transition region with the axisymmetric flow regime. As the complexity of the flow increased the period of the vacillation cycles grew to $\sim 100-180$ 'days'. This slower vacillation seemed to involve a cyclic enabling and disabling of nonlinear interactions between the forced stationary wave and the growing and azimuthally drifting wave, which in turn was linked to a decrease in mean flow shear. Subsequent chains of wave-wave interactions characterised the complex but robust oscillation phenomenon. The resulting behaviour has several features in common with some recent models of intraseasonal oscillations in the mid-latitude troposphere and with sudden stratospheric warmings.
\end{abstract}

Keywords: Baroclinic instability; Baroclinic waves; Sloping convection; Topography; Flow regimes

\section{Introduction}

In a companion paper (Read and Risch (2011), hereafter Paper I), we presented results from a new laboratory study of the interaction between thermally-driven, rotating baroclinic flow and periodic bottom topography. In that paper, the main features of the apparatus and methodology were described, and a new regime diagram was presented which shows more clearly and comprehensively than in previous studies the rich variety of complex flows which can be exhibited in the presence of topographic forcing at a single azimuthal wavenumber (in this case, $m=3$ ). The results of that paper noted the applicability of the Charney-Drazin theory for large-scale wave propagation in a rotating, stratified fluid, which helped to account for the organization of the regimes observed in parameter space, including the occurrence of largeamplitude stationary waves which penetrate to all heights in the apparatus. We also found that multi-stable flow states were common throughout the wave-dominated regimes, though this was not necessarily greatly enhanced compared with flows without bottom topography.

*Corresponding author. Email: p.read1@physics.ox.ac.uk 


\subsection{Low-frequency variability in the atmosphere and oceans}

Another important motivation for the present experiments arises from an interest in mechanisms for low-frequency variability in baroclinically unstable systems which only entail internal linear or nonlinear dynamics, as opposed to variations of external forcing or boundary conditions. There is an ongoing debate in the climate research community concerning the relative importance of processes involving either external cyclic forcing in the atmosphere (e.g. due to the annual cycle or processes in the ocean circulation) or nonlinear, autonomous internal variability.

James and James (1989), James and James (1992), and James et al. (1994), for example, found evidence for spontaneous variability on timescales from months to years (or even decades) in simple GCM experiments with constant forcing conditions, which appear to be due entirely to internal chaotic processes within the model atmosphere itself. This has lead to significant uncertainty in the interpretation of a variety of phenomena identified in climate observations and numerical simulations, including the North Atlantic Oscillation (NAO) on timescales of tens of days (sometimes known as the 'Index Cycle', Wallace (2000)) to several months or years, and various 'Intraseasonal Oscillations' such as the Madden-Julian oscillation in the tropical atmosphere (ref: Madden \& Julian 1994) and comparable oscillations in the extra-tropics (e.g. Branstator 1987, Ghil 1987, Kushnir 1987, Dickey et al. 1991). In several of these phenomena, the role of topographic forcing has been suggested as having an important dynamical role in inducing oscillatory behaviour on timescales of greater than a few tens of days (e.g. Jin and Ghil (1990)).

In the stratosphere, too, a number of sources of variability are generally regarded as owing their origin to topographic forcing at the Earth's surface, such as those responsible for the episodic breakdown of the winter polar vortex leading to Stratospheric Sudden Warmings (SSWs - e.g. Andrews et al. (1987)). The mechanism for SSWs is commonly understood to arise from the resonant breaking of large-scale Rossby waves, forced by the interaction of the underlying flow with continent-scale topographic features (e.g. Matsuno 1971), though the nature of this breakdown and the extent to which it may be dependent on seasonal changes in the large-scale circulation remain controversial (O'Neill and Pope 1988, Charlton and Polvani 2007, Charlton et al. 2007, Matthewman et al. 2009). In particular, the possible origin of SSWs and their variability as intrinsic instabilities (e.g. Yoden 1987, Esler and Scott 2005), or due to external influences (e.g. Scott et al. 2008) remains uncertain with possible roles suggested either for resonant effects with topographically forced waves (Plumb 1981) or smaller scale phenomena (Birner and Williams 2008).

The occurrence of SSWs is also not necessarily confined solely to the Earth, but there is some evidence from numerical simulations that a dynamically similar phenomenon may occur in the Martian atmosphere (Barnes and Hollingsworth 1987, Wilson 1997, Kuroda et al. 2009), where the amplitude of continent-scale topography is larger even than for the Earth.

\subsection{Low-frequency variability in the laboratory}

Examples of low-frequency oscillations have also been reported in the literature for a number of years for rotating annulus experiments, both with and without topography (e.g. Buzyna et al. (1978), Bernardet et al. (1990) and Früh and Read (1997), respectively).

In the absence of bottom topography, low frequency variability is observed to occur as the result of long-timescale vacillations of wave amplitude and/or structure. Within the regular wave regimes in the rotating annulus, Buzyna et al. (1978) found slow and chaotic variability associated with modulations of the applied thermal contrast between the sidewalls. The frequency of the associated variability, however, was not directly commensurate with the frequency of the modulation of the boundary conditions. Read et al. (1992) and Früh and Read 
(1997) found evidence for such slow vacillations in association with the amplitude vacillation phenomenon and related bifurcations, in which the amplitude of a baroclinic wave grows and decays periodically via interactions with the zonal mean flow. The period of the amplitude vacillation itself was consistent with a dependence mainly on Taylor number, such that the period diverged towards very large values (in apparent association with a homoclinic bifurcation) at a critical value of $\mathcal{T}$. By varing thermal Rossby number, however, Read et al. (1992) and Früh and Read (1997) also found that the quasi-periodic amplitude vacillation would also undergo a bifurcation involving the spatial sidebands of the dominant wavenumber, leading to a mode competition in which the amplitude vacillation itself could undergo a cyclic (though often chaotic) modulation in time, on a timescale which could also diverge without limit as a particular point in parameter space was approached, in the form of a 'soft mode instability'.

For the case where topography was present, Bernardet et al. (1990) conducted experiments with radially non-varying wave-2 topography and a free surface at five selected fixed points in parameter space, of which two cases coincided with the region covered in the present study. These two cases were characterised by low-frequency vacillations of the dominant drifting wave- 6 amplitude on a time-scale which corresponded to about 50 annulus revolutions. The period of one vacillation cycle was observed to be equal to the time it took wave-6 (and wave-4, which was found to have the same drift-frequency) to drift by one wave-length. The interaction was therefore interpreted as a constructive and destructive interference between the drifting waves and the stationary wave-2. Similar results had been reported earlier by Leach (1981) and Li et al. (1986).

Bernardet et al. (1990) suggested a possible analogy of some of their observed features to the 30-60 day oscillation found in the NH extra-tropical atmosphere (e.g. Anderson and Rosen 1983, Jin and Ghil 1990)). Such an analogy would suggest that the mechanisms for the atmospheric oscillation could be governed entirely by internal dynamics of the mid-latitude atmosphere, and that forcing, say from the tropics or oceans, is not essential for it to occur. In this context it may be of some interest to note that the scale separation between the drifting baroclinic waves and the stationary wave-2 in Bernadet et al.'s experiments was similar to that generally associated with the mid-latitude storm tracks on Earth, which have a planetaryscale structure, upon which smaller-scale baroclinic waves grow and decay. It would also be of interest to investigate whether or not a similar low-frequency phenomenon occurs when the horizontal scale of the topography is similar to the scale of the transient waves themselves. This is the case in the present experiments, and also in the atmosphere of Mars (e.g. Hollingsworth et al. 1996) where storm tracks can be identified during the winter seasons in each hemisphere but the dominant wavenumber of both transient and stationary waves is typically $m=1-3$.

In a previously sparsely explored region of parameter space (see Paper I), the present experiments have also demonstrated the existence of a robust oscillation - subject to hysteresis - of significantly lower frequency (100-180 annulus revolutions) than those observed by Bernardet et al. (1990) (around 30-60 annulus revolutions). These new observations in our experiments raise questions over possible alternative analogues in the atmosphere on a longer timescale, comparable with intraseasonal variations and even the seasonal cycle itself. In this paper, some possible similarities between the laboratory flows and atmospheric phenomena such as intraseasonal oscillations and major stratospheric sudden warmings (SSWs) are examined. Such global-scale SSW events are only generally observed to occur once per winter (but even then not every year), and also only in the northern hemisphere, where land-sea thermal contrasts and orography produce more intense stationary waves than in the southern hemisphere. The nature of SSWs continues to excite controversy, with debates continuing over whether they occur because of vortex interactions with the winter polar vortex or because of wave-zonal flow mechanisms. Such an analogy as discussed here would shed valuable insight into the possibility that SSWs are part of a cyclic vacillation phenomenon in the atmosphere due to interactions between topographically forced and internal planetary waves, but which 
is periodically interrupted by large changes in the thermal forcing during the seasonal cycle.

\subsection{Outline}

This paper is organised as follows: Section 2 briefly outlines the laboratory apparatus and analysis methods used in the present study (though the main details are presented in Paper I). Results from the experiments are presented in section 3 and 4, analyzing in detail two distinct kinds of low-frequency phenomena found in different regions of parameter space. Section 5 summarises and discusses the results in terms of the new insights compared with earlier laboratory studies and in terms of their possible relevance to causes of slow variability of the atmospheric general circulation on Earth and Mars.

\section{Apparatus and Methods}

The laboratory system under investigation was described in some detail in Paper I, and so we only give a brief outline of the essential features here. The apparatus used was essentially the same thermally-driven rotating annulus as used in studies by Hignett et al. (1985) and Hignett (1985), though with improved instrumentation and control systems as described in Paper I. A fluid is contained between two upright, coaxial brass cylinders of radii 2.5 and 8.0 $\mathrm{cm}$, and between horizontal, thermally-insulating boundaries at heights above the bottom of zero and $14.0 \mathrm{~cm}$. Topography was introduced in the form of a sinusoidally-varying false base, with wavenumber $m=3$ structure in azimuth, no variation with radius, and an amplitude of $1.1 \mathrm{~cm}$.

The temperature of each boundary was controlled independently to a precision of around $\pm 0.02 \mathrm{~K}$, and the outer cylinder is typically maintained at a higher temperature than the inner. The entire system was then rotated at angular velocity $\Omega$ (with a stability $\sim 1$ part in $10^{4}$ ), where $\Omega$ ranged in the present experiments from around $1-3 \mathrm{rad} \mathrm{s}^{-1}$. This enabled the system to operate over a range in the principal nondimensional parameters:

$$
\begin{aligned}
& \mathcal{T}=\frac{4 \Omega^{2}(b-a)^{5}}{\nu^{2} d}, \\
& \Theta=\frac{g \alpha \Delta T d}{\Omega^{2}(b-a)^{2}},
\end{aligned}
$$

where $\mathcal{T}$ is the Taylor number and $\Theta$ the stability or Thermal Rossby number, $a$ and $b$ are the inner and outer radii, $d$ is the depth of the annulus, $\Delta T$ is the temperature difference between the outer and inner wall, $\nu$ is the kinematic viscosity and $\alpha$ is the coefficient of thermal expansion. The parameters used for all the experiments discussed here and in Paper I are listed in Appendix A below. The temperature contrast $\Delta T$ and rotation rate $\Omega$ were under continuous computer control, enabling these parameters to be varied either continuously in time or in sequences of short steps, as well as being capable of being maintained at constant values. Such controlled variations are invaluable in enabling large regions of parameter space to be explored semi-automatically. However, caution is sometimes necessary in the interpretation of the results of such experiments, especially with continuously varying scanning, since this can lead in some cases to behavior that is induced directly by the parameter variations (e.g. see Buzyna et al. 1978, Bishop and Galvanetto 1993, Hua and Lu 2001, Kogan 2007).

Measurements were routinely made of the temperature of the walls and other parts of the apparatus at intervals of $20 \mathrm{~s}$ throughout each run using copper-constantan thermocouples. The flow itself was visualised and measured at up to 5 levels in the vertical using a suspension 


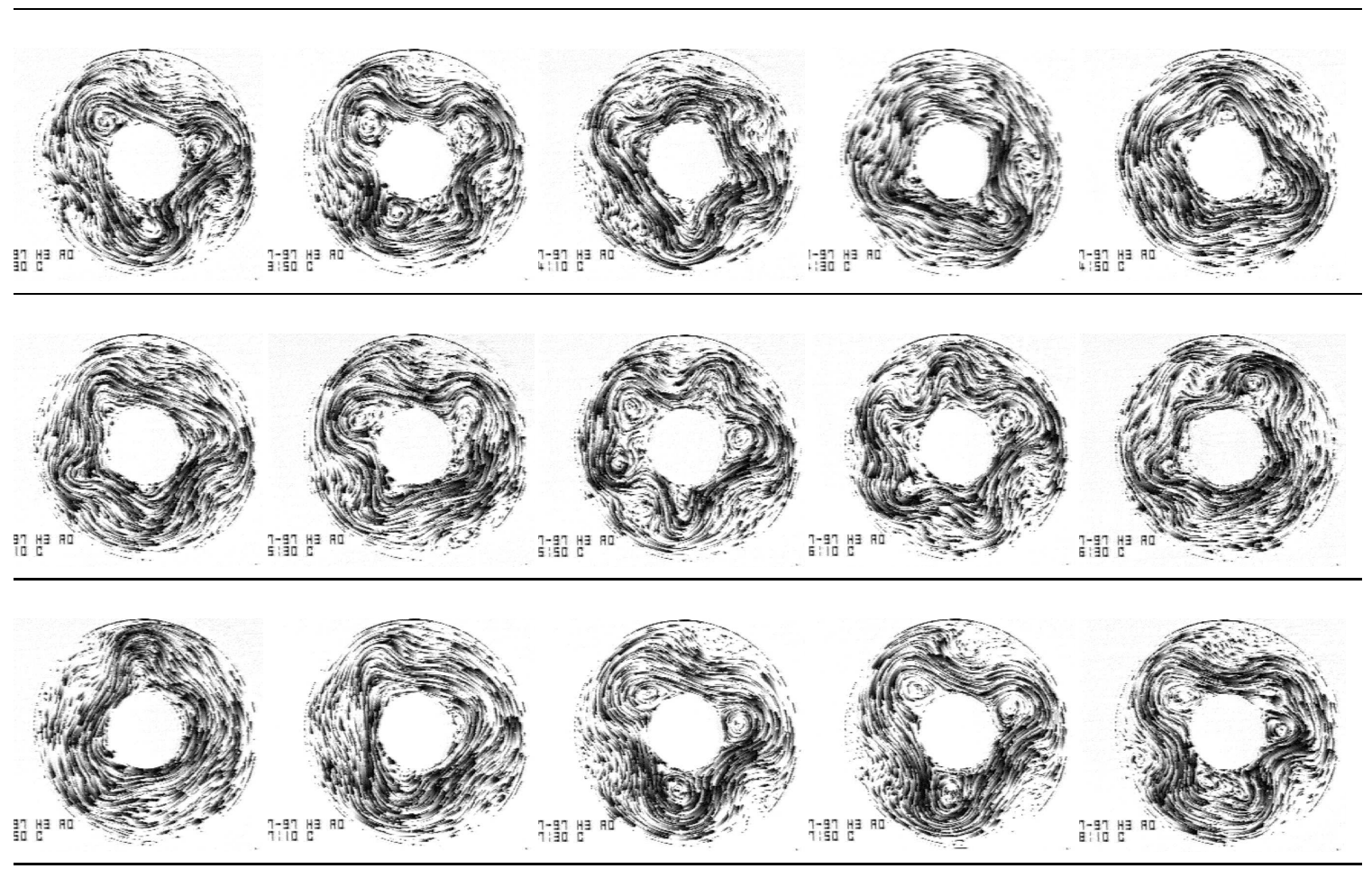

Figure 1. Sequence of streak movie snapshots (20s apart) at level 2 showing the decay and re-establishment of a stationary wave-3 over a 5 minute interval. The sequence runs from left to right and top to bottom. The plot corresponds to $B 3 r$ at $\mathcal{T}=24 \times 10^{6}, \Theta=0.17$.

of neutrally-buoyant Pliolite particles of diameter $\sim 300 \mu \mathrm{m}$, illuminated from the side via perspex windows in the outer sidewall and viewed from above in the rotating frame via a CCTV video camera. Visualisation was achieved by continuously tracking particles illuminated at a chosen level and displaying streaks accumulated over 2.5 - 10s. Such streak images could be displayed either in real time or recorded on a time-lapse video recorder. Continuous image sequences of particle motions (recorded on SVHS videotape) could also be analysed using DigImage software to obtain quantitative velocity fields either at single level or cycled through several levels over a period of up to $30 \mathrm{~s}$. More details of the analysis procedure can be found in Paper I.

\section{Stationary wave amplitude vacillation (SAV)}

\subsection{Flow variability}

Over most of the drifting wave parameter regime the amplitude and structural variability of the dominant drifting wave modes appears to be very complex. A Fourier time-series analysis, however, reveals that a relatively regular or periodic amplitude vacillation of the stationary wave-3 component underlies most flows. In the SAV-region of parameter space (see figure 7 of Paper I) a quasi-stationary wave-3 temporarily grows to large enough amplitudes such that it dominates and can also be identified on streak movies. The periods of wave- 3 domination are characterised by a quickly forming wave-3 pattern whose (eastward) drift slows down over a few tens of degrees until it becomes almost stationary. At this point its amplitude grows to a maximum until it begins to drift eastwards again and decays.

The improved flow visualisation allows the naked eye to follow in great detail the evolution of the quasi-periodic but complex SAV patterns, giving a good first intuitive picture. As an example, figure 1 shows a sequence of streak movie snapshots at level 2, each 20 s apart, which 

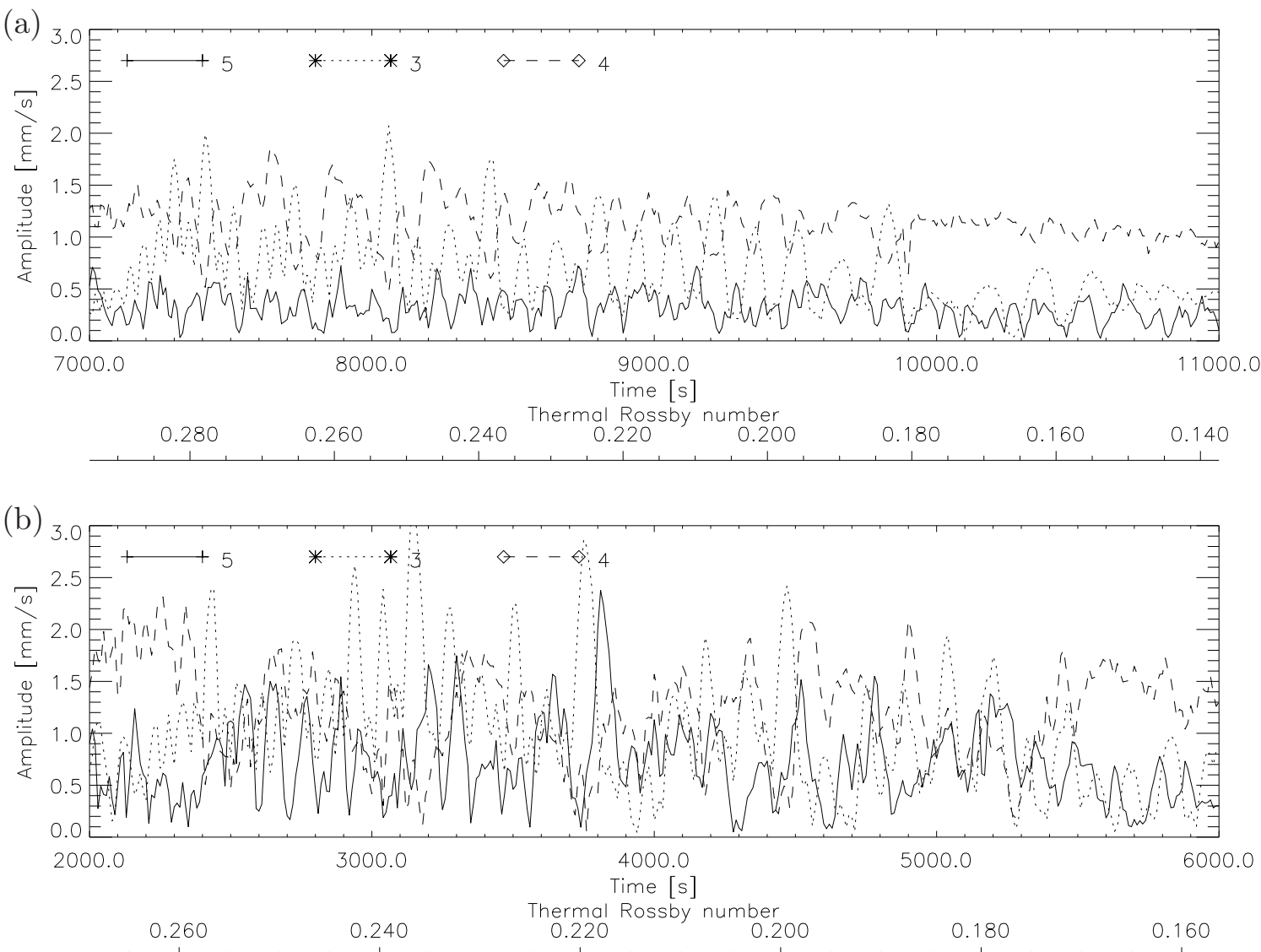

Figure 2. Time-dependent amplitudes of waves 3,4 and 5 during SAV at level 2 mid-radius, (a) for B1 $\left(\mathcal{T}=12.3 \times 10^{6}\right)$ and (b) for B3 $\left(\mathcal{T}=20.6 \times 10^{6}\right)$.

covers just over two 'cycles' of a wave-3 decay and growth. It illustrates the strong stationary wave- 3 competing with a structurally very distorted wave- 4 and also a temporary dominance of a wave-5. The subsequent almost total decay of the drifting waves then leads into the growth of another quasi-stationary wave- 3 which eventually starts to evolve into a drifting mode again, this time directly into a wave- 5 .

Figure 1 was produced from a 5 minute interval of experiment B3, in which $\Delta T$ was steadily decreased over the range $14.5-1.9 \mathrm{~K}$ while keeping $\Omega$ fixed (see Table A, reproduced from Paper I). This experiment generally has very similar characteristics to B1. In order to investigate the effect of $\mathcal{T}$ on the variability characteristics of the flow regimes encountered by B1, experiment B3 scans the $\mathcal{T}-\Theta$ parameter space vertically at level 2 in the same manner as B1 by linearly decreasing $\Delta \mathrm{T}$ while keeping all other parameters constant; $\mathcal{T}=20.6 \times 10^{6}$ in B3 compared to $\mathcal{T}=12.3 \times 10^{6}$ in B1. It generally crosses the same types of flow regime as B1 (see figure 5 of Paper I). Small differences include a slightly larger parametric extent of dominating wave-2 in B3 followed by a slightly smaller extent of wave- 4 domination. As in B1, the subsequent transition at similar values of $\Theta$ to a SAV flow is followed by another wave- 4 domain before a quasi-steady stationary wave-3 flow is established.

For a similar time-span and range of $\Theta$, figure 2 compares the SAV flow encountered in B3 with that of B1 in terms of the amplitudes of the most dominant modes, i.e. wave-3, 4 and 5. For clarity the phases are not shown in figure 2 but instead are presented over a shorter interval in figure 3 (noting that azimuthal phase is defined herein as increasing towards the "west"). The most prominent feature of the wave-3 phase is the quasi-stationarity which coincides with each wave-3 amplitude maximum, similar to figure 10 of Paper I. The most 
notable difference between B1 and B3 is a generally more structurally noisy wave-field in B3. Specifically the SAV cycles occasionally include a dominating wave-5 mode in B3 which seems to disorganise the temporal behaviour quite substantially. For example, a fairly robust feature of figure $2 \mathrm{a}$ is the periodicity of the dominance of wave- 3 which recurs every time the wave has drifted by 3 wavelengths, i.e. once around the annulus, between 7700 and 9200s. The wave- 5 component, although not insignificant, never grows enough to dominate. Figure $2 \mathrm{~b}$ on the other hand shows a very irregular appearance of wave- 3 dominance. Rather than peaking once every time the drifting component has advanced by $6 \pi$, it dominates the flow after a fairly random but integer number of $2 \pi$ phase drift periods. Occasionally it becomes dominant after several successive $2 \pi$ phase cycles (2900-3300s). The growth of a strong and sometimes dominating wave- 5 signal is often connected with an exceptionally large-amplitude wave-3 peak (e.g. 3200s, 3850s, 4500s). This suggests that the wave-5 draws its energy from a wavewave as opposed to a wave-mean flow interaction.

The important point is that there seems to be a temporally regular underlying wave-3 amplitude vacillation throughout the SAV regime. The above examples demonstrate how this important dynamical feature may not always be easily identifiable from the streak movies, despite their high quality. It is important to bear in mind that the timeseries shown in figures 1-3 involve a smooth change in the external parameter $\Theta$ and are not taken at a fixed point in parameter space (such as e.g. in Bernardet et al. (1990)). The complexity of the temporal evolution of the wave-patterns might be expected to be enhanced by the variation in $\Theta$. Even so, a characteristic flow evolution defines the SAV regime, the parametric boundaries of which agree well in terms of $\Theta$ between experiments B1 and B3, despite significant differences in $\mathcal{T}$.

\subsection{Wave-3 resonance}

The lower boundary of the SAV regime deserves some more attention in order to clarify what the underlying nature of the vacillation might be. The existence of a quasi-steady wave-4 regime at values of $\Theta$ below the SAV regime and above the region of parameter space which is totally dominated by a stationary wave- 3 is suggested by figure 5 of Paper I and figure 2 . In figure 5 of Paper I, the coincidence of the disappearance of a measurable drifting wave3 component with the vanishing of the SAV phenomenon strongly suggests that resonance between the drifting and stationary components is a crucial element in the mechanism for the large intermittent growth of wave-3. It is also observed that SAV involves a fast wave3 decay after a sharp amplitude maximum, which is typical for a flow which temporarily satisfies a resonance condition. If a stationary wave- 3 were to grow gradually to a point where it dominates the flow, there is no reason to believe that it should decay again in the SAV parameter domain. Evidence for this is provided by experiments B7i and D2i which cover similar regions of parameter space but vary $\Omega$ at a constant value of $\Delta T$; see Table A. These experiments support a quasi-steady, stationary wave-3 throughout the domain where SAV has been observed.

Hysteresis was observed in association with a number of the flow transitions, as noted in Paper I, and made it difficult to define boundaries of some flow regimes precisely on a regime diagram, particularly for the drifting wave-3 and SAV regimes. Although B1 and B3 agree very closely on the values of $\Theta$ between which SAV occurs, lower values of $\Theta$ were still found to support SAV if the flow regime was approached from a different direction. This applies both to B7r and D2r which exhibit SAV at values of $\Theta$ as low as 0.155 . This raises the question as to whether the SAV is associated with a drifting wave-3 regime after all or whether it is only an overlapping zone between the stationary wave- 3 and the drifting wave- 4 regimes with random intermittent switching between the two. In other words, doubts may arise as to whether the existence of a region of quasi-steady wave- 4 between the SAV and stationary wave- 3 regimes is merely a result of the (relatively fast) decrease in the forcing parameter $\Theta$ 
(a)

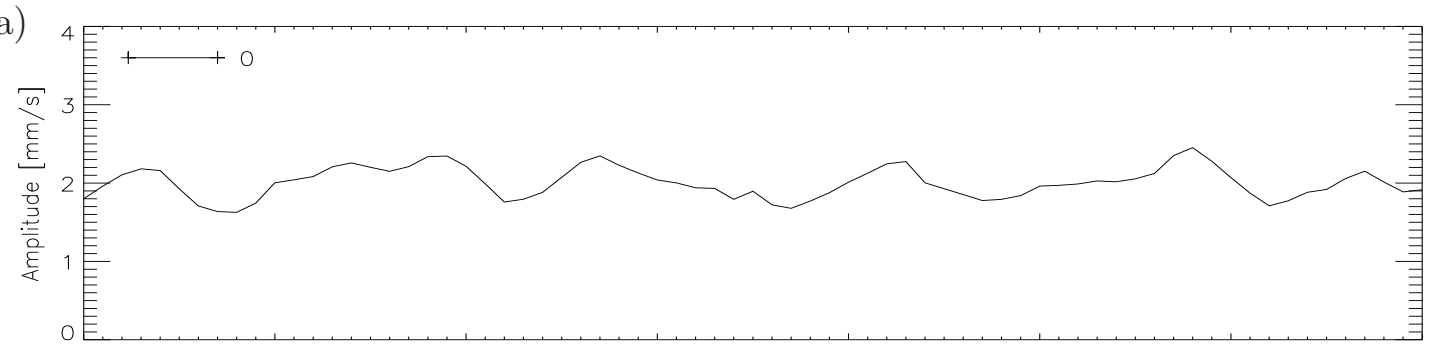

(b)

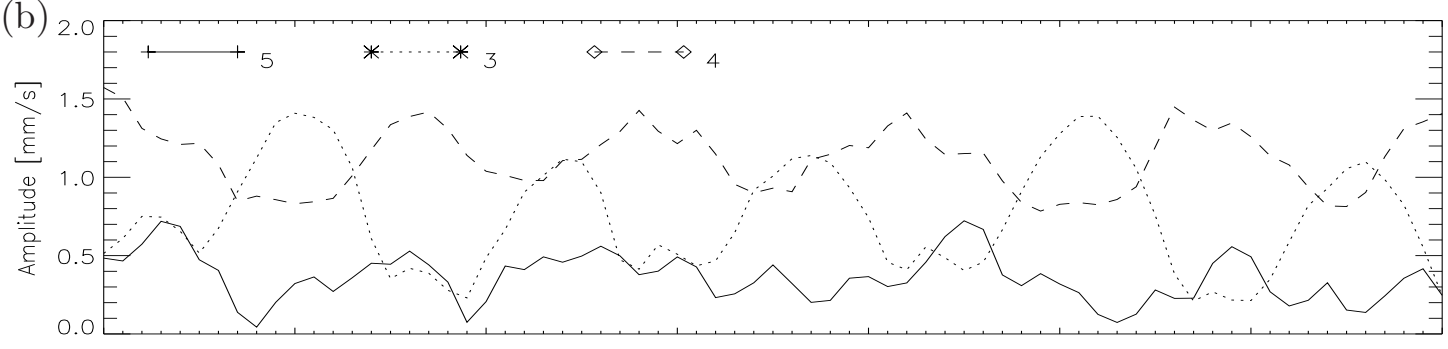

(c)

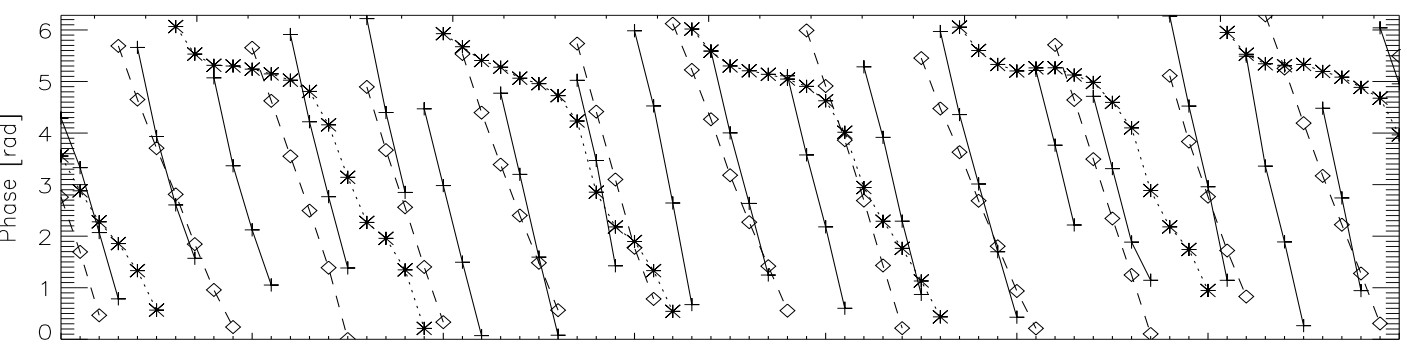

(d)

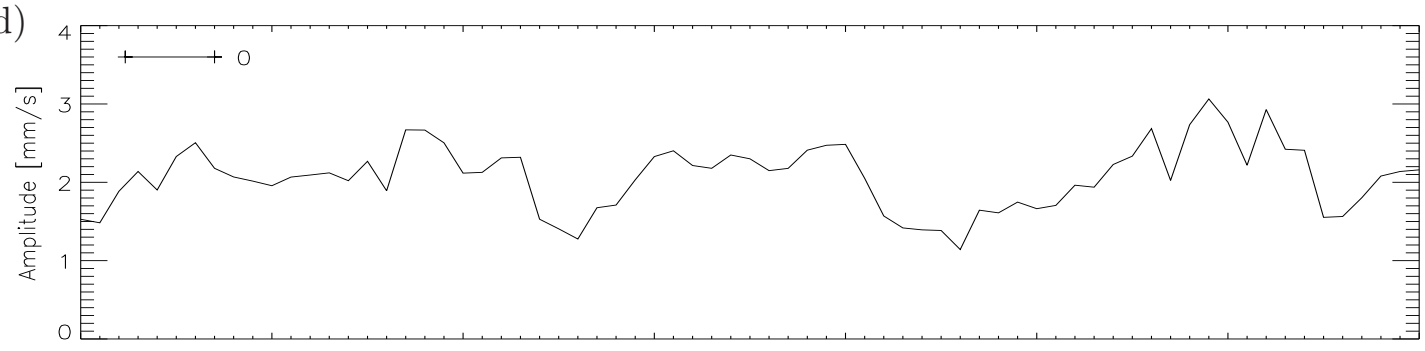

(e)

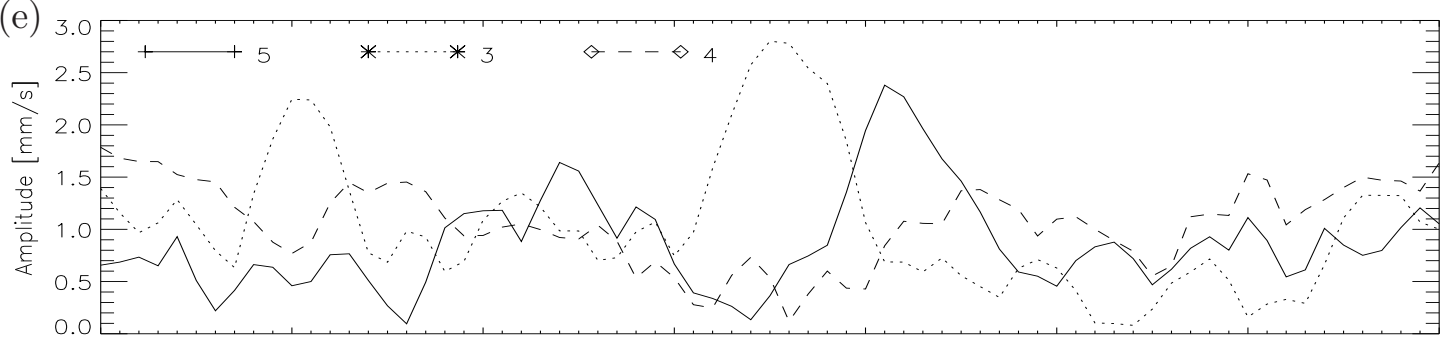

(f)

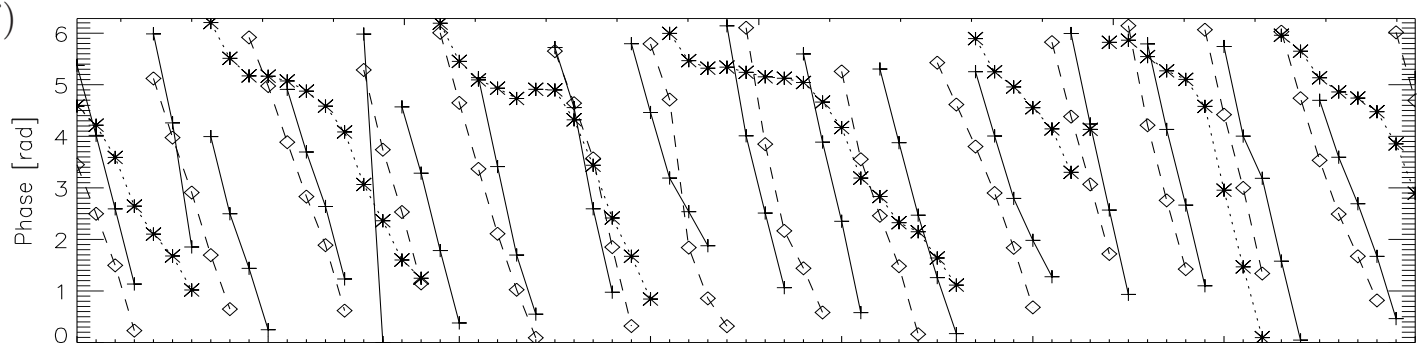

Figure 3. Time-series of amplitudes of waves $0,3,4$ and 5 during SAV at level 2 mid-radius, $\Theta \sim 0.22$; (a-c) for B1 at $\mathcal{T}=12.3 \times 10^{6} ;(\mathrm{d}-\mathrm{f})$ for $\mathrm{B} 3$ at $\mathcal{T}=20.6 \times 10^{6}$. 

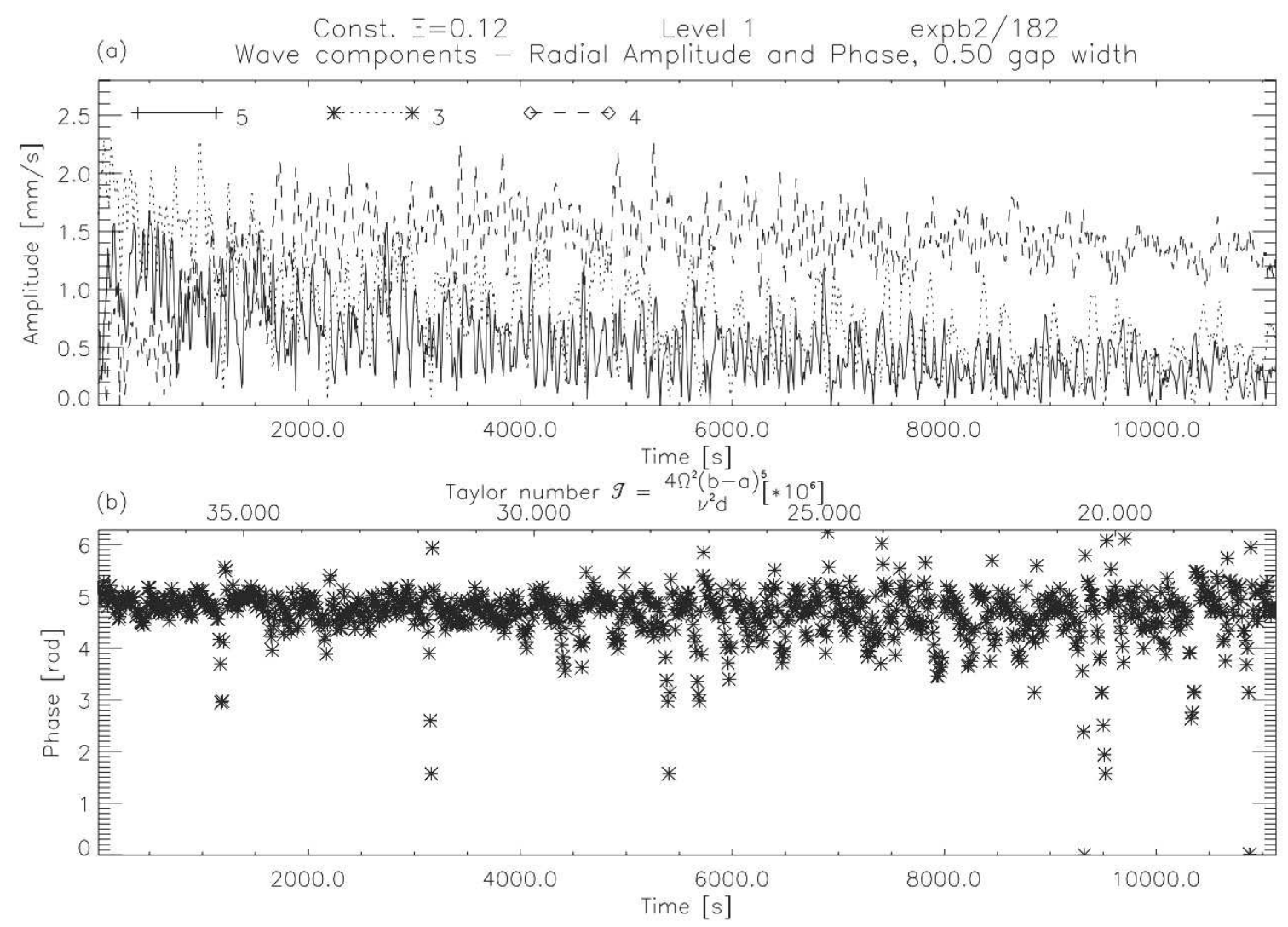

Figure 4. Timeseries of D3r $(\Theta=0.12=$ const. $)$ at level 1 mid-radius showing (a) the amplitudes of waves 5,3 and 4 , and (b) the phase of wave-3.

during B1, B3 and B7.

In order to test the robustness of this part of the wave- 4 regime, experiment D3 was conducted (see Table A). It is similar to D2 in that it approaches the region of interest by smoothly varying $\mathcal{T}$. The constant value of $\Theta$ for D3 was chosen to lie lower than the lowest $\Theta$ where SAV had been observed, and just above those values where a transition to the stationary wave-3 occurred in B1, B3 and B7. Quantitative data were collected during the first 3 hours of the scan beyond which qualitative flow visualisation was continued up to the point where the flow pattern changed to a stationary wave-3. As figure 4 shows, the steady wave- 4 , though erratic, clearly dominates throughout almost the entire scan. Any measurable drifting wave-3 components do not normally drift by a whole wave-length and remain insignificant compared to the stationary component.

It seems, therefore, that the SAV phenomenon is associated with a distinct regime in which a drifting wave- 3 component interacts with the forced stationary wave- 3 in a manner suggestive of a topographic resonance phenomenon. This could entail resonant triad nonlinear wave-wave and wave-zonal flow interactions (given oscillations and variability in the mean zonal flow are observed as well as variations in the waves themselves) that involve a number of different wave modes. This is explored further below in the context of the LAV regime, and in a companion series of papers by Marshall and Read (2015). The period of vacillation corresponds to the drift period of wave-3 consistent with a nonlinear interference between drifting and stationary wave components. 
Similar observations had been made in previous work. For example, Bernardet et al. (1990) and Pfeffer et al. (1990) performed experiments with a differentially heated rotating annulus with wave- 2 bottom topography at a fixed point in parameter space corresponding to $\mathcal{T}=2.1 \times$ $10^{7}$ and $\Theta=0.24$, which lies within the SAV regime in our experiments. Their observations of low frequency variability were similar in character to SAV in the sense that they observed a cyclic growth and decay of a wave component on time-scales corresponding to $\sim 30-60$ annulus revolutions. Bernadet et al. interpreted their measurements as a drifting wave amplitude vacillation, since in their experiment a drifting wave- 6 was both dominant and vacillating regularly in amplitude every time the wave- 6 had drifted by one wave-length. In this sense it may be perhaps more appropriate to refer to SAV as a drifting wave amplitude vacillation. However, since the streak movies only allowed the identification of a wave-3 pattern near its maximum amplitude, which corresponds to the quasi-stationary phase of the cycle, the wave-3 was interpreted primarily as a topographically forced stationary wave.

\section{Low frequency amplitude vacillation (LAV)}

In addition to SAV, the regime scans also identified a region of parameter space at slightly larger $\Theta$ and smaller $\mathcal{T}$ than the SAV regime (but within a similar range of $\Delta T \geq 4 K$ ) where a robust, quasi-periodic variability phenomenon was found. This turned out to be the most regular, lowest frequency and largest amplitude vacillation over the entire region of parameter space investigated. The LAV regime itself may be divided into a region at lower $\mathcal{T}$, where a relatively simple wave amplitude vacillation was found which was dominated by wave-4, and a more complex type of LAV at higher $\mathcal{T}$ which involved interactions with more wave-number components. The vacillation periods ranged from about 100 annulus revolutions for the 'simple' LAV to 180 for the 'complex' LAV. Each flow will now be described in turn.

\subsection{Simple $L A V$}

Figure 5 shows a sequence of streak movie snapshots during LAV, alternately switching between levels 4 and 2 every 20s. It corresponds to the third LAV cycle from the left in figure 6a, which in turn corresponds to experiment D1r near the point in parameter space where D1 and $\mathrm{C} 5$ cross $\left(\mathcal{T} \sim 8.95 \times 10^{6}, \Theta=0.44\right)$. The difference in streak lengths in figure 5 gives an impression of the relatively slow (mainly eastward) velocities at level 4 compared to level 2. It also shows a tendency to minimise this difference during the wave- 4 amplitude peaks. More importantly perhaps, the streaks at level 4 reveal a detectable amount of wave-activity throughout the whole LAV cycle, even if this is only in the form of small eddies near the inner wall, suggesting that wave-generation mainly occurs near the bottom.

Figure 6a reveals that the LAV in this parameter region is very regular and periodic and dominated by a wave- 4 . A slight gradual decrease in the wave- 4 amplitude maxima with decreasing $\mathcal{T}$ is noticeable. A wave-2 signal, whose phase is drifting (not shown), is present during or just after the decay of wave-4, but is of relatively small amplitude. The significance of the wave- 2 will become more apparent in the discussion below of the more complex LAV at higher $\mathcal{T}$. No detailed analysis of the phase propagation at level 2 and 4 is presented here since the temporal resolution was not sufficiently high.

In order to follow the evolution of the LAV with further decrease of $\mathcal{T}$, another 'window' of quantitative data was obtained at $\mathcal{T} \sim 5.15 \times 10^{6}$. The wave amplitudes at level 2 are shown for comparison in figure $6 \mathrm{~b}$. Notice that the y-axis range is half of that in figure 6a. With time and decreasing $\mathcal{T}$, the smaller wave amplitude maxima continue to diminish towards a state where it would become indistinguishable from a quasi-steady wave-4, as observed in the streak movies. The drifting wave- 2 and wave- 6 signals seen in figure $6 a$ have virtually 

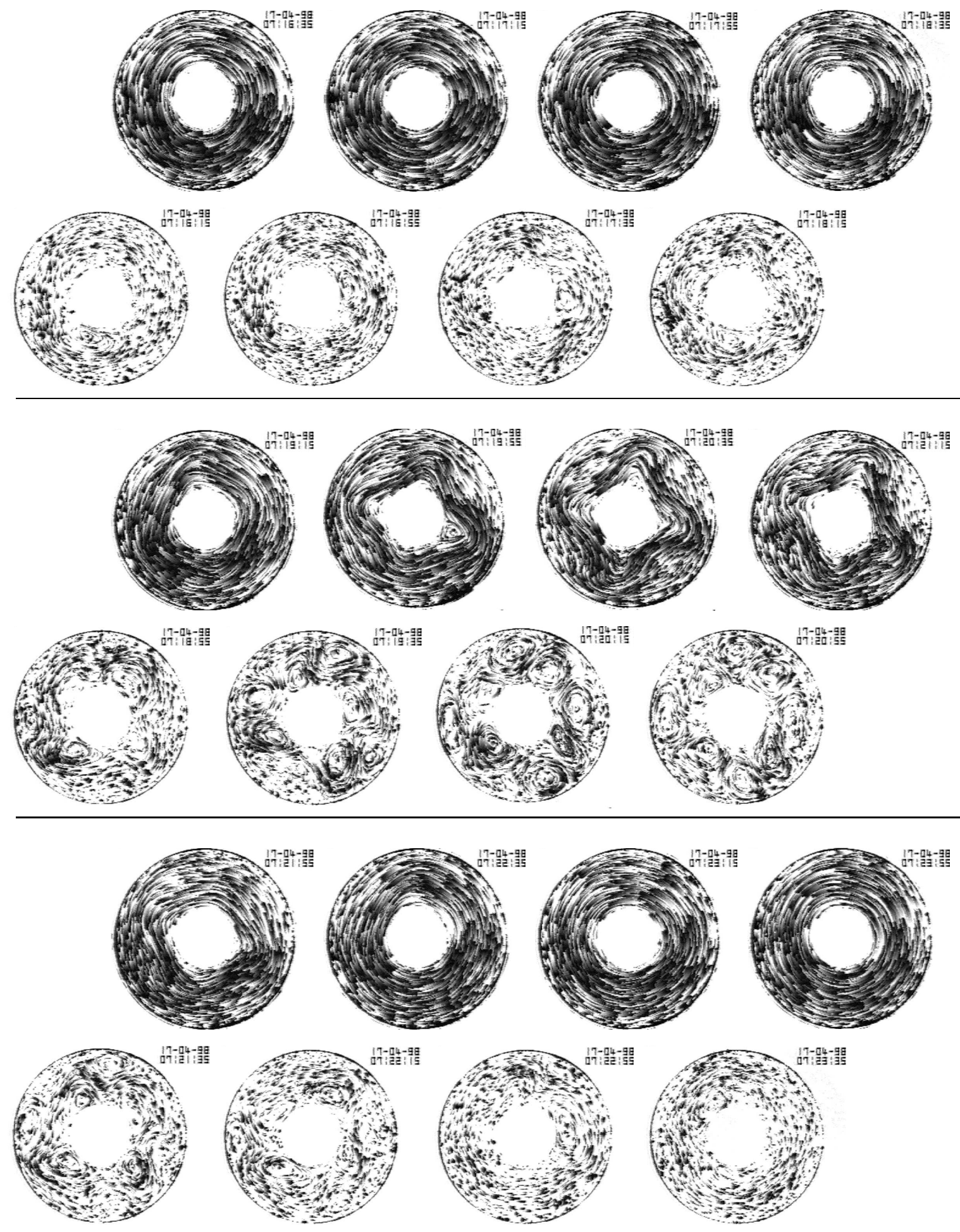

Figure 5. Sequence of streak movie snapshots (20s apart) showing the growth and decay of a wave- 4 for about $85 \%$ of one cycle of low frequency amplitude vacillation (LAV) over a $7 \mathrm{~min} 40 \mathrm{~s}$ interval. The sequence runs from left to right and top to bottom with images alternating between level 4 (lower row) and 2 (upper row). The corresponding experiment and location in parameter space were $\mathrm{D} 1 \mathrm{r}$ and $\mathcal{T} \sim 8.95 \times 10^{6}, \Theta=0.44$. 

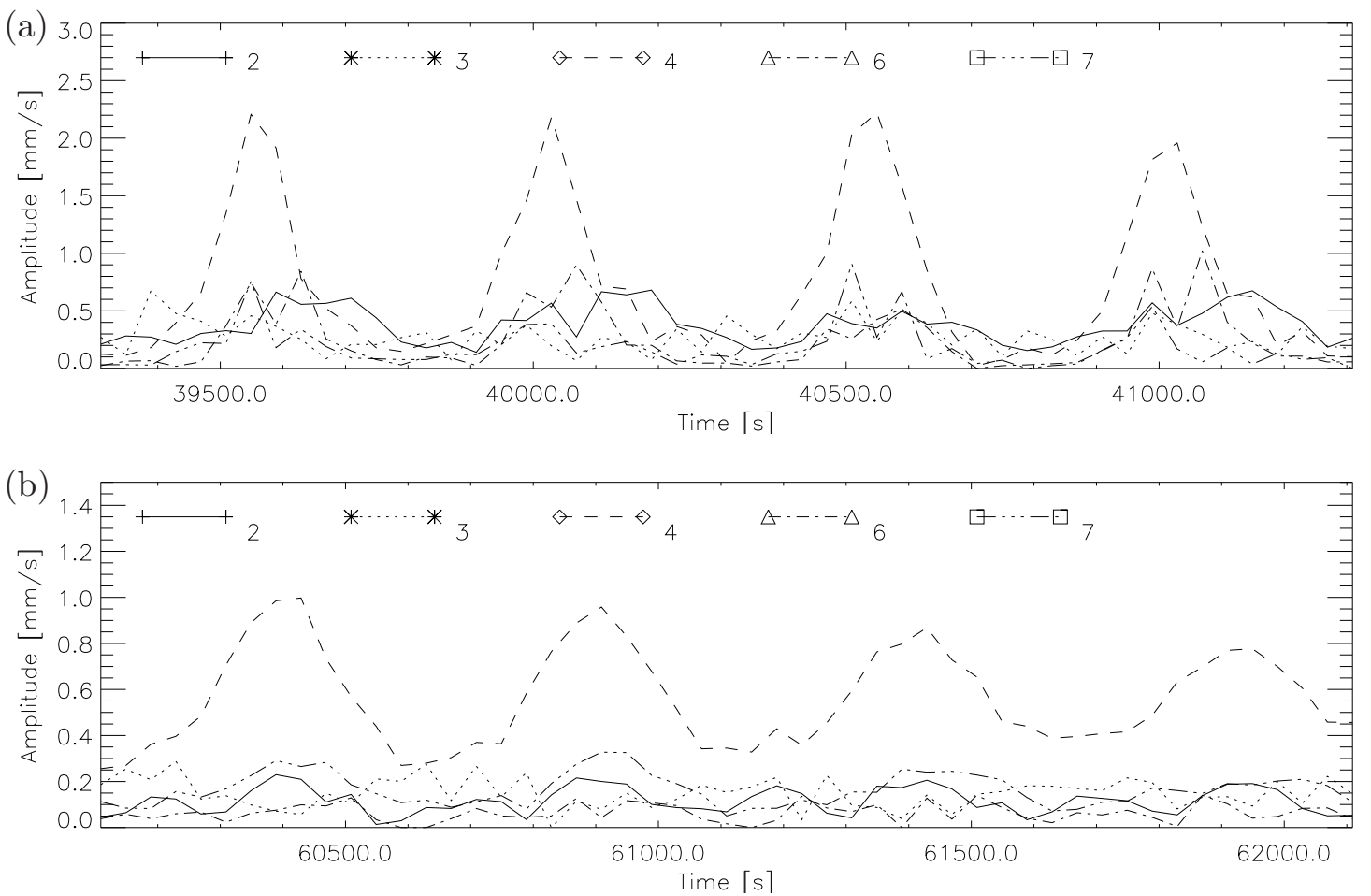

Figure 6. Time-series of wave-amplitudes at level 2 mid-radius over a 2000s interval, comparing (a) D1r around $\mathcal{T} \sim 8.95 \times 10^{6}$ and (b) D1r around $\mathcal{T} \sim 5.15 \times 10^{6} ; \Theta=0.44$ (held constant); note the differing y-axis scales.

disappeared. An important observation is that the LAV still persists regardless of the fewer significant wave-numbers and the lower wave-amplitudes. Furthermore, the period of about 500 s is exactly the same as that of figure 6a. Additional confirmation of the independence of the LAV period on $\mathcal{T}$ comes from D1i which again adjusted to the same LAV period as in D1r after the initiating abrupt flow disturbance. This result implies that the period of LAV depends directly on the thermal Rossby number $\Theta$.

\subsubsection{Resonant triads}

The main feature of the observations at low $\mathcal{T}$ lies in the relative simplicity of the LAV characteristics. It enables the identification of some of the underlying dynamics of the more complicated LAV-phenomenon seen at higher $\mathcal{T}$ and $\Theta$. Due to the relatively slow drift-speed of the waves, the phase propagation is also sufficiently well resolved for quantitative analysis at low $\mathcal{T}$. Figure 7 thus shows quantitative comparisons of the most prominent wave amplitudes and phases between level 2 and 4 . One feature of a likely fundamental dynamical mechanism can be seen in figure $7 \mathrm{~b}$. The frequencies of wave- 4 and wave- 7 are, on average, the same, i.e. $\omega_{k=4}=\omega_{k=7}$. This phenomenon, referred to as phase-locking, is highly suggestive of a nonlinear interaction. Due to the presence of wave-3 topography, a likely scenario would be a resonant interaction between a stationary wave- 3 and drifting waves 4 and 7 which dominate the flow. The necessary conditions for resonance, i.e.

$$
k_{1} \pm k_{2} \pm k_{3}=0 \quad \text { and } \quad \omega_{1} \pm \omega_{2} \pm \omega_{3}=0
$$

are both satisfied.

But a closer look at figures $7 \mathrm{~b}$ and d reveals that the second condition is only satisfied over part of the cycle; that part corresponding to a growth in wave- 4 amplitude. During the other part of the LAV cycle a drifting component of wave- 3 is present and the phase differences between wave- 4 and 7 are not constant. The fact that the resonance condition is satisfied 

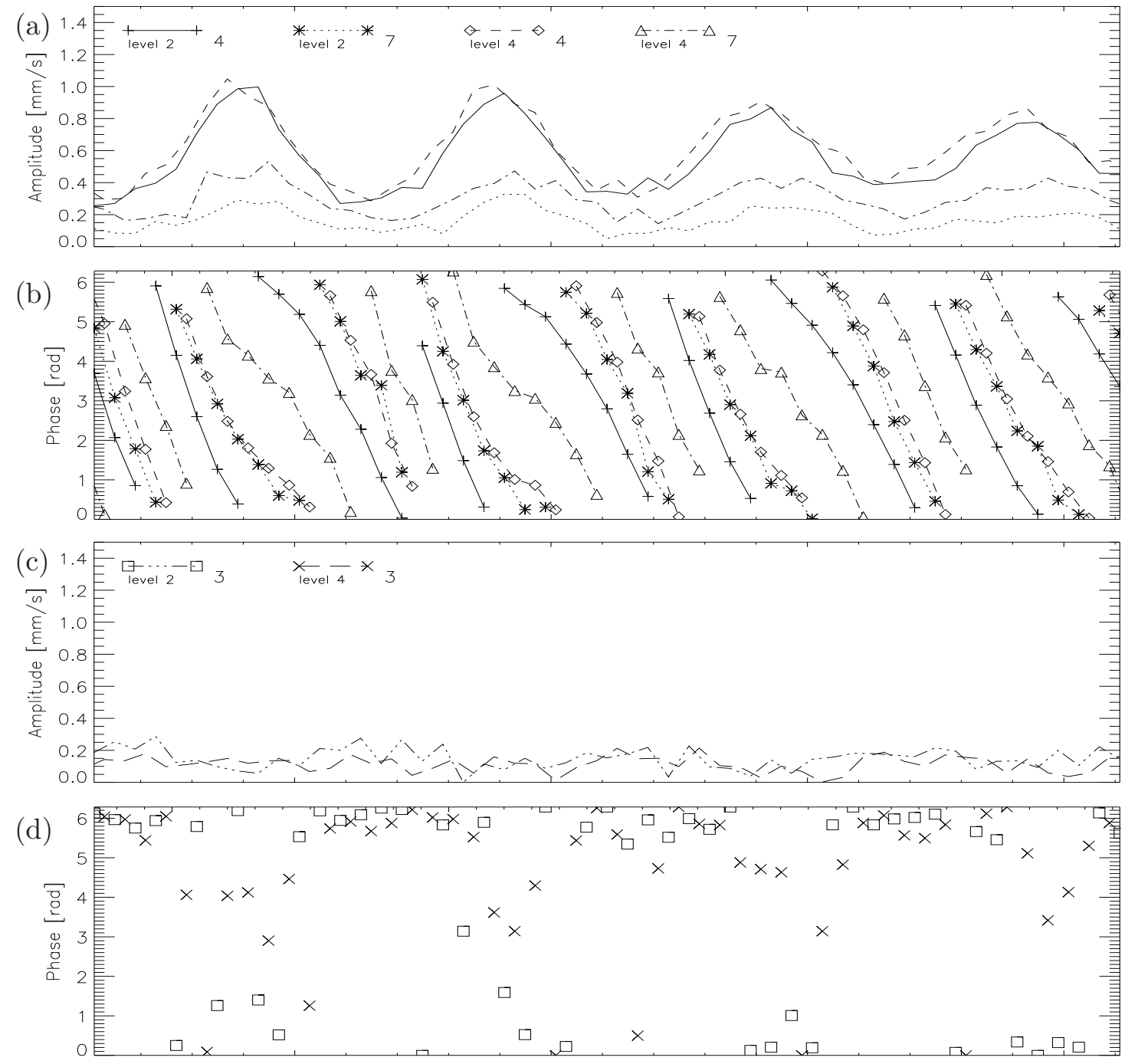

(e)

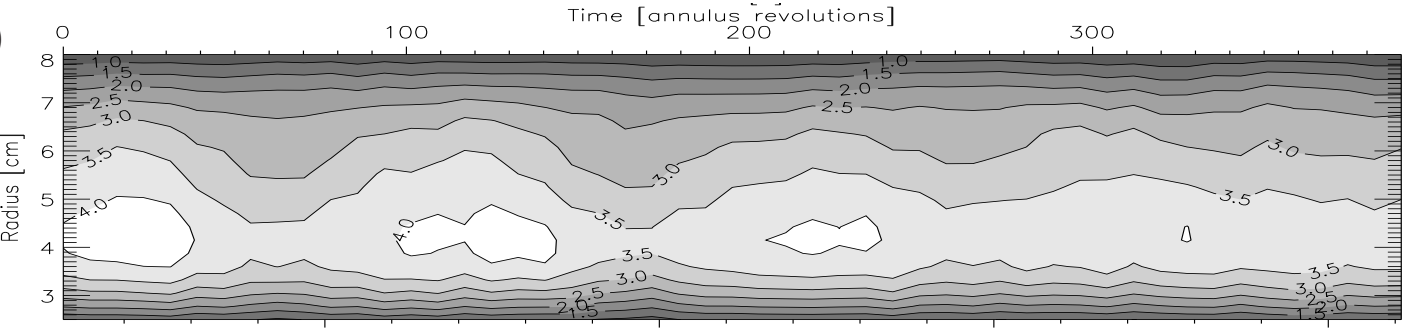

(f)

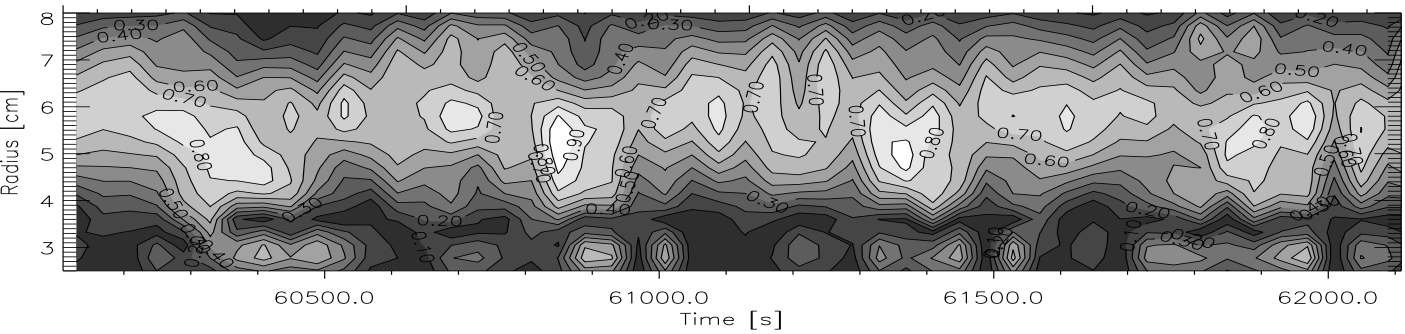

Figure 7. Time-series of wave-amplitudes, phases and mean flow at level 2 and 4 during D1r $\left(\mathcal{T} \sim 5.15 \times 10^{6}, \Theta=0.44\right)$; (a) wave-amplitudes at mid-radius of wave 4 and 7 at level 2 and 4 , (b) phases of waves 4 and 7 , (c) amplitudes of wave 3 , (d) phases of wave 3, (e) zonal flow (wave 0) at level 2, (f) zonal flow (wave 0) at level 4. 
during the wave- 4 growth period suggests that energy is transferred into wave-4, although the origin of this energy is not clear. Simple considerations might suggest that energy is provided from the forced wave-3 component. This is partly consistent with detailed analyses conducted, for example, by McEwan et al. (1972) who studied numerically the behaviour of three resonant waves, of which one wave was directly forced. When the forced wave amplitude exceeded a critical value, energy was transferred to the other two modes such that a steady amplitude of the two unforced modes was established and the amplitude of the forced wave remained at the critical value. This is consistent with the present observations of an apparently limiting amplitude of the forced wave-3 (figure 7c), which, though not necessarily constant, does not vary significantly or in any regular fashion, particularly at level 4 . The growth of the wave- 4 in this context would then be a consequence of an ever stronger wave- 3 forcing due to an increasing strength of zonal flow over the topography, which would be justifiable for the level 4 (but not for level 2). The numerical experiments by McEwan et al. (1972) also suggested, however, that, unless the mode with the highest frequency was forced, all waves except the forced one would decay. Furthermore, evidence from numerical experiments by Fjørtoft (1953) suggest that the energy transfer would have to occur simultaneously to both larger and smaller scales.

This would not strictly apply to the present situation where wave-3 has zero frequency on average (although a back and forth azimuthal movement around its mean stationary position is noticeable) and where the energy transfer is primarily to smaller scales (waves 4 and 7). The notion in the present context that two (or more) drifting wave modes of comparatively smaller scale tend to extract energy from a forced stationary wave component, however, is not new. The laboratory investigation of wave triads by Jonas (1981) noted a similar behaviour, although no definite principle or mechanism for this effect could be established. But overall, it seems unlikely that a single triad resonance, forced by a stationary wave- 3 which loses energy to wave- 4 and wave-7, is sufficient to account for what is observed in these experiments.

In practice, it seems more likely that more than one resonant wave triad contributes to the dynamical exchanges of energy within the flow. A wave- 4 component, for example, could grow in amplitude spontaneously through baroclinic instability and interact with a topographically forced wave-3 to form a resonant triad with wave-7. The resonance condition on frequencies (see Eq (3)) would then be consistent with wave- 4 and wave-7 having the same frequency and with Fjørtoft's requirement for wave- 4 to lose energy to both higher and lower wavenumber. But other wave-wave interactions are also likely to be involved. This is more clearly apparent in the more complex LAV flows discussed below, but may also be present in the simpler flows too. Similar kinds of interaction involving nonlinear interference effects between different wavenumber components, either stationary or drifting, and the mean zonal flow were also discussed by Buzyna et al. (1989), Ohlsen and Hart (1989) and Pfeffer et al. (1990) in the context respectively of wave amplitude and zonal flow vacillations without topography and baroclinic wave interactions with periodic topography. This is explored further in the work presented in the companion paper by Marshall and Read (2015).

A radial cross-section of zonal mean zonal flow at level 2 is shown in figure 7e. The mean zonal flow minima occur during or just after the wave- 4 amplitude maxima, while the mean flow maxima occur during the first half of the wave- 4 growth. In comparison, the mean flow at level 4 shown in figure $7 \mathrm{f}$ (notice the different contour levels) does not seem to follow the same pattern. In fact, the maximum zonal flow at level 4 coincides with the maximum wave- 4 amplitude at that level, i.e. almost coincident or just prior to the mean flow minimum at level 2. Since the zonal flow maxima at level 4 tend to occur at relatively small radii compared with the jet location at other times they could be interpreted as being connected with the evolution of the zonal flow at level 2. The maximum of the latter occurs a few tens of seconds earlier than the level 4 maximum but at similar or only slightly smaller radii. The mean flow acceleration at level 2 is most likely to be directly due to the thermal forcing, while the mean 
flow increase at level 4 seems to be more subtly connected with an adjustment of the vertical shear in the mean flow.

Finally, another feature of figure $7 \mathrm{~b}$ is that the phases at level 4 slightly lag behind those at level 2 for waves 4 and 7 . This implies an eastward phase tilt with height in radial velocity which, somewhat surprisingly, would normally suggest baroclinic decay rather than growth and instability. The tilt is greatest around the amplitude maxima and more pronounced for wave-7. The phases of wave- 3 on the other hand, as indicated in figure $7 \mathrm{~d}$, do not show a clear phase difference between level 2 and 4, particularly during the stationary phase, which implies a nearly barotropic structure. Other wave-features, which are not shown on the grounds that the wave-amplitude is below a threshold of about $0.2 \mathrm{~mm} / \mathrm{s}$, include a quasi-stationary wave-2 and wave- 1 , and a drifting wave- 6 which was typically phase-locked to wave- 4 and wave- 7 .

\subsection{Constant amplitude drifting wave-4}

Experiment D1i (see Table A) was characterised by the formation of a drifting wave- 4 which persisted despite a continuous increase in $\mathcal{T}$ into the LAV regime, analogous to C1i where $\Theta$ was smoothly increased. At the upper levels the wave- 4 pattern was very weak except near the inner wall. A very high temporal resolution timeseries at level 4 revealed that the flow was in a quasi-equilibrium state exhibiting some interesting nonlinear dynamics. This involved phaselocking between wave- 4 and 7 and hence most likely a wave-3-4-7 triad interaction, similar to the wave- 4 amplification mechanism of the LAV cycle. Figure 8 shows that the wave4 and 7 amplitudes and phase-differences are remarkably constant. This suggests that the wave-wave interaction process itself is spontaneous and due to the presence of the stationary component of wave-3, i.e. it has no significant inherent temporal variability, even though the wave-3 fluctuates back and forth around its average position on a time-scale of a drift period of wave- 4 or 7 , as shown in figure $8 \mathrm{~b}$. Notice the much shorter time-scale of the wave-period as compared with figure 7 (300s for 8 periods versus 2000s). This may be partly due to the slightly higher values of $\mathcal{T}$ and thus a stronger mean flow, but for the most part it is due to the lack of LAV. The main conclusion from this is that the wave-3-4-7 triad interaction by itself cannot account for the LAV phenomenon. Since the magnitude of the constant wave- 4 amplitude in figure $8 \mathrm{a}$ can be estimated to lie roughly between the minimum and maximum amplitudes of the LAV cycle at this point in parameter space (the latter may be estimated by interpolating between figure 6a and b), it may be regarded as an 'equilibrium' around which the LAV flow oscillates. Due to the robustness of the LAV flow as a result of the positive feedback mechanism, however, it may be more appropriate to interpret the quasi-steady flow as a meta-stable state which might only require a small perturbation to 'kick' it into an oscillating LAV mode.

The mean flow variation across the channel is shown in figure $8 \mathrm{c}$ with the same contours as figure $7 \mathrm{f}$. The location of the mean flow maxima at around $5 \mathrm{~cm}$ radius are very similar. Figure $8 \mathrm{c}$ does not, however, show a displacement of the jet to larger radii between the maxima. Instead, the amplitude of the mean flow increases significantly at very small radii $(\sim 3 \mathrm{~cm})$. Figure 9a reveals that the direction of the flow at small and large radii is westward. Interestingly the overall pattern differs significantly from a regular, stationary wave- 3 pattern, more so than in figure $9 \mathrm{~b}$ which is shown for comparison with the same contour intervals and corresponds to the LAV cycles of figure 7 . This may be due to an enhanced stationary wave-2 or wave-1 activity which might result from a nonlinear interaction with the forced stationary wave-3.

Although, as mentioned above, it is not entirely clear how energy gets transferred between various wave-components, the possible transfer of energy to the stationary wave- 2 and wave- 1 components would provide a mechanism of energy transfer to larger scales, while the wave-3-47 resonance would transfer some energy to smaller scales. Thus, the Fjørtoft (1953) condition 

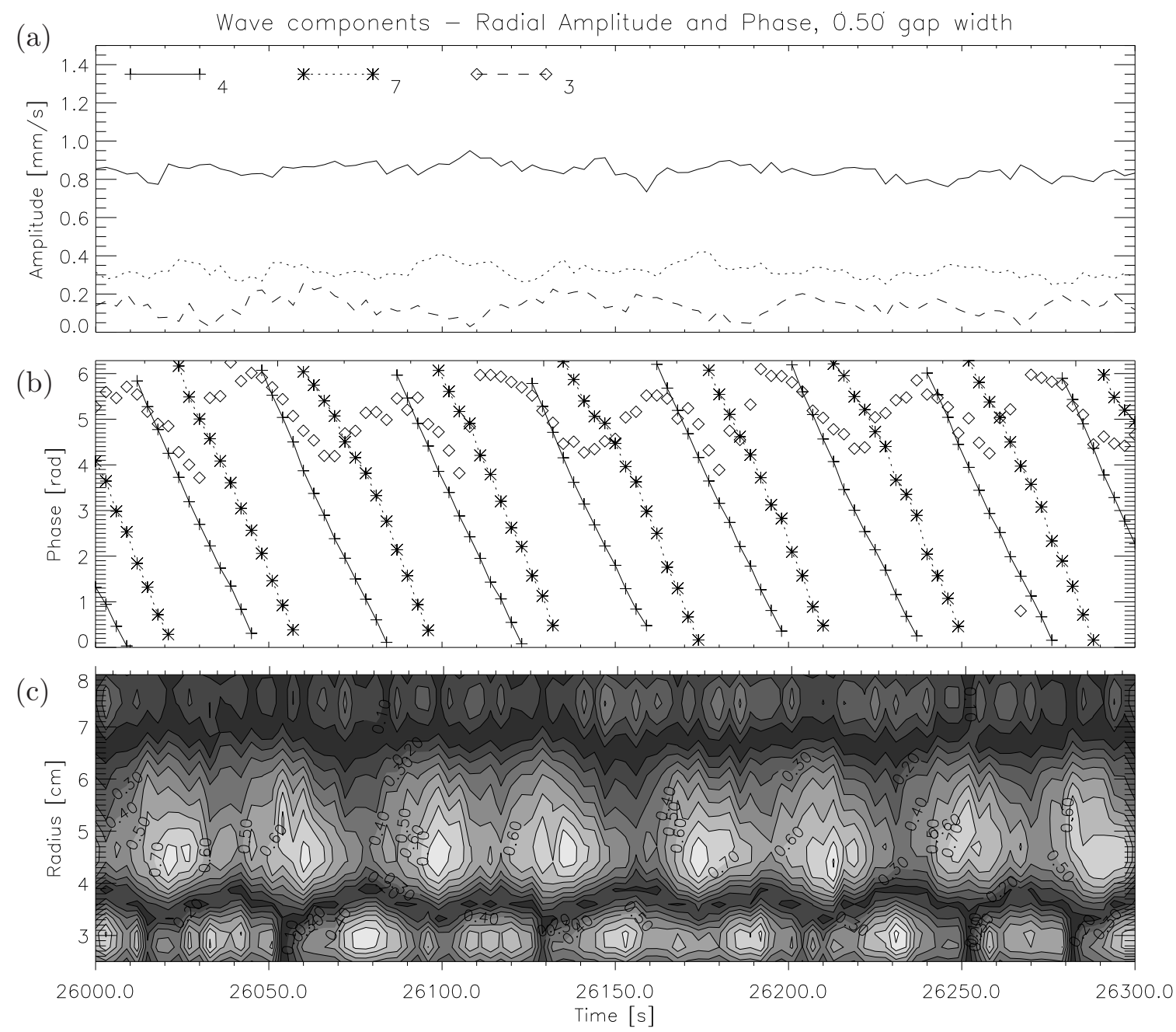

Figure 8. Time-series of D1i at $\mathcal{T} \sim 6.00 \times 10^{6}(\Theta=0.44)$ at level 4 showing (a) amplitude of waves 4,7 and 3 at mid-radius and (b) phase of wave- 4 , wave- 7 and wave- 3 at mid-radius and (c) zonal mean zonal flow variation across the gap.

(a) Time-averaged Zonal velocity
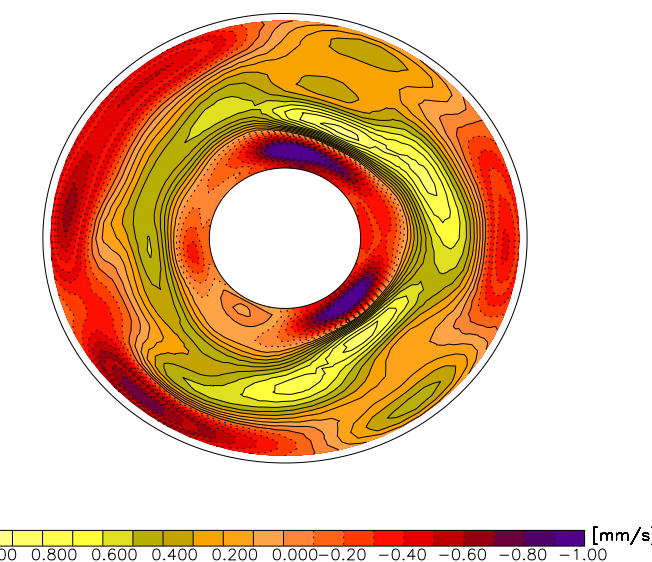

(b)

Time-averaged Zonal velocity

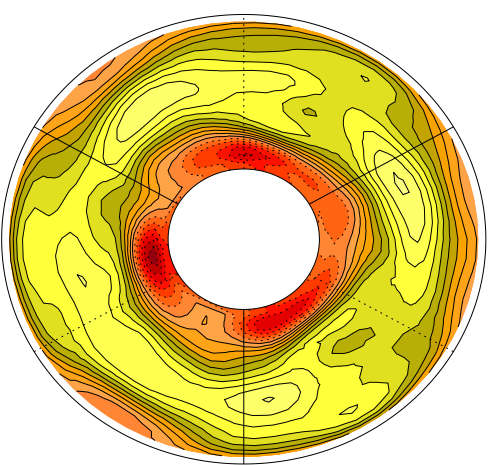

$[\mathrm{mm} / \mathrm{s}]$

Figure 9. (colour) Time-mean zonal velocity over 8 wave drift cycles at level 4 for (a) D1i at $\mathcal{T} \sim 6.00 \times 10^{6}$ and (b) D1r at $\mathcal{T} \sim 5.15 \times 10^{6} ; \Theta=0.44$ 


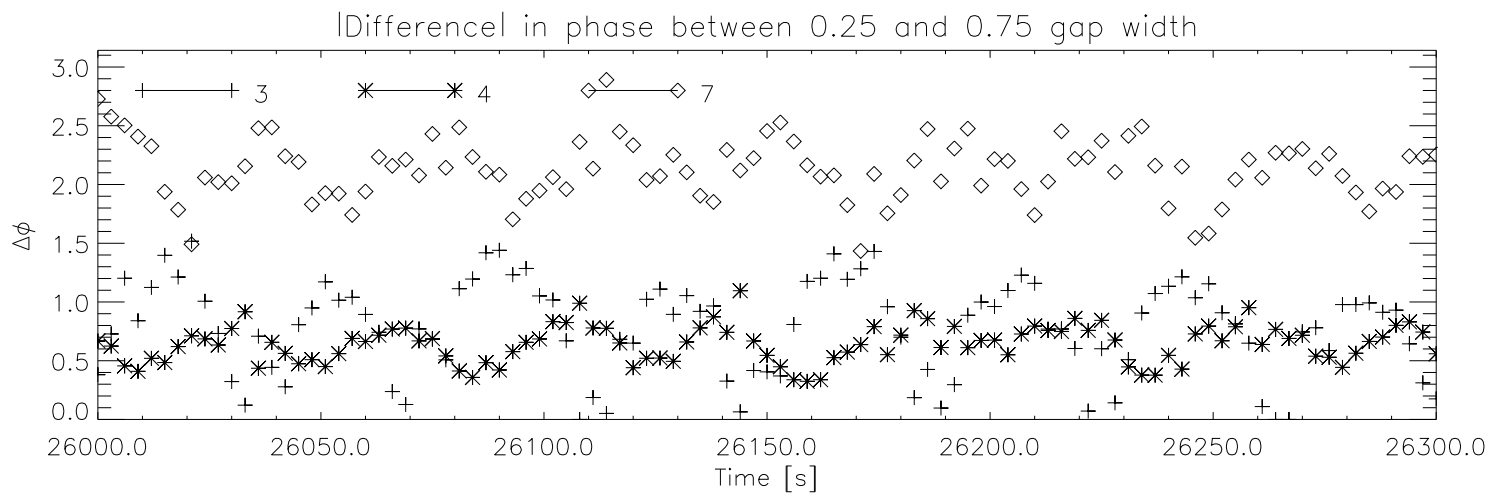

Figure 10. Time-series for D1i at $\mathcal{T} \sim 6.00 \times 10^{6}$ at level 4 showing the phase difference between $1 / 4$ and $3 / 4$ gap width for each wave.

for wave resonance, which requires simultaneous transfer of energy to both larger and smaller scales, may be met in the context of nonlinear interactions involving more than just one triad interaction in the present experiments.

\subsubsection{Radial wave components}

Equation 3 expresses the necessary conditions for resonance for the wave-generating and decaying mechanisms suggested by the observations in terms of the zonal wave-number $\mathrm{k}$ and the frequency $\omega$. Full justification for interpreting a process as a wave-triad interaction, however, further requires that the radial wave-numbers (l) also separately add up to zero. A possible scenario which would fulfil this criterion would be

$$
(3,1) \leftrightharpoons(4,1) \leftrightharpoons(7,2)
$$

where $(k, l)$ denote zonal and radial wave-numbers and the arrows show possible directions of energy flow such that one wave supplies energy for the growth of the other two waves.

The criterion for the radial wave components was tested by comparing the phase at $1 / 4$ gap width to that at $3 / 4$ gap width. A radial wave- 1 would imply zero phase difference while a radial wave- 2 would require a phase difference of (ideally) $\pi$. Figure 10 shows the phase difference between the waves at $1 / 4$ and $3 / 4$ gap width which correspond to figure 8 . A relatively small phase difference across the channel is seen for waves 3 and 4 . Wave-7, on the other hand, has a phase-difference across the channel on average $\sim 2.0$. The fact that it is not equal to $\pi$ may be explained by the fact that, in reality, many more wave components are present which may interact with the dominant waves. A similar method of identifying radial wave components will be applied to the more complex LAV flows below. A distinction between a radial wave- 2 and wave- 1 will then be made on the basis of whether the phase difference between 0.25 and 0.75 gap-width is greater or less than $\pi / 2$, respectively.

\subsection{Complex LAV flows}

Perhaps the most intriguing observations of LAV were made during the first 3 hours of experiment $\mathrm{B} 9 \mathrm{r}$ for which quantitative data is available (see Table $\mathrm{A}$ ). Upon decreasing $\Delta \mathrm{T}$ in small regular steps every 2400 s, the flow evolved from exhibiting oscillations at the lowest observed frequencies, which involved strong wave- 2 and wave- 4 components, to a flow dominated mainly by a wave- 4 oscillation of slightly higher frequency. The timespan of the quasi-periodic amplitude vacillation cycles ranged from just over 700s to about 500s over the 3 hour period. 
(a)

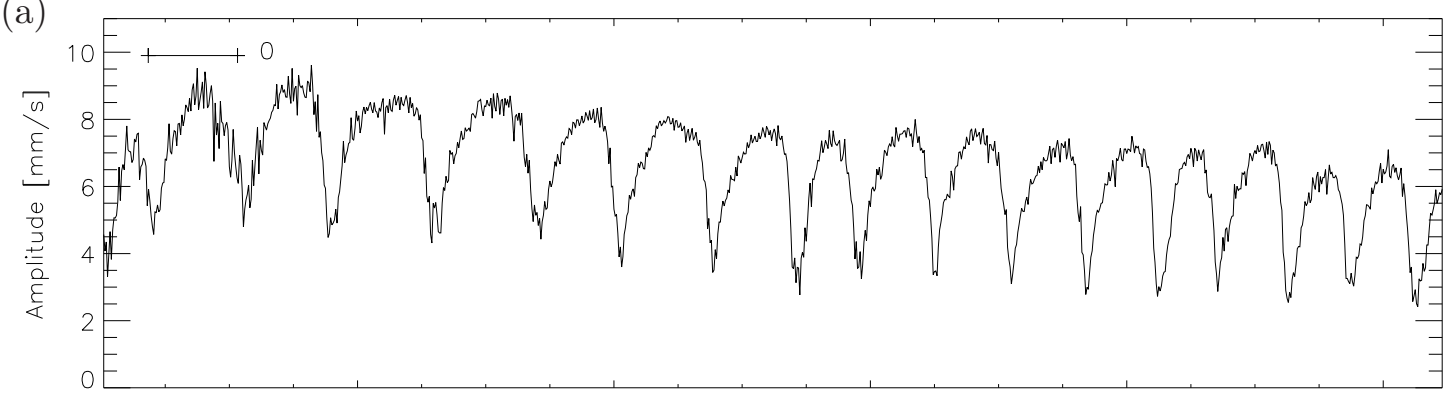

(b)

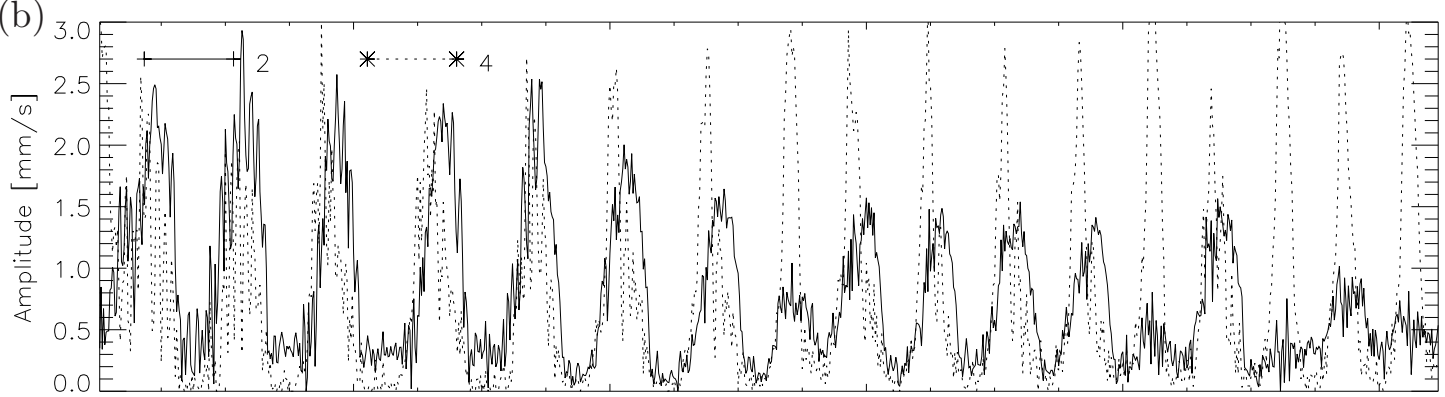

(c)

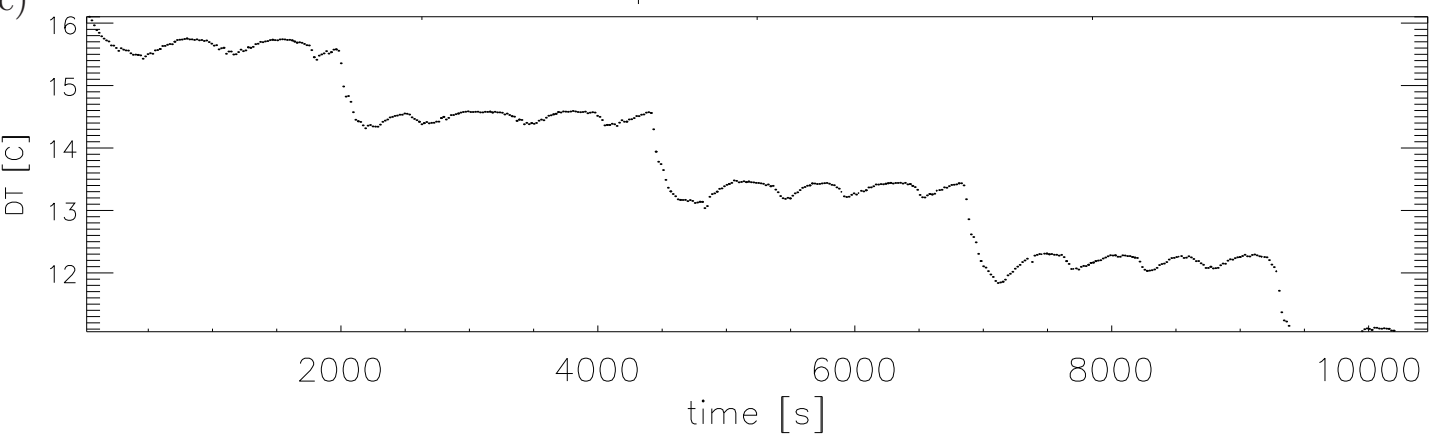

Figure 11. Time-series of B9r $\left(\mathcal{T}=10.71 \times 10^{6}=\right.$ const.) at level 1 mid-radius showing (a) Zonal velocity amplitude (wave 0), (b) Radial velocity amplitude of wave 2 and 4, (c) Measured wall temperature difference.

This corresponds to about 180 to 130 annulus revolutions. Figure 11b shows the corresponding radial velocity wave amplitude time-series for waves 2 and 4 at level 1 . The peaks of the wave- 4 amplitude bursts are comparatively steep and narrow while those of wave- 2 are broader and delayed with respect to wave-4. The combined strong wave-activity coincides with a sharp drop in zonal mean flow amplitude (wave-0), as a comparison with figure 11a shows. Characteristic for this time series is also the fact that no two vacillation cycles are the same and the period of each cycle is generally longer for larger wave- 2 amplitude maxima. Of course the variation of the control parameter $\Delta \mathrm{T}$ has some effect on the flow's variability. But as a comparison with figure 11c shows, the amplitude vacillation feature is robust against fairly sudden changes in $\Delta \mathrm{T}$ (at 2000, 4500, 7000 and $9030 \mathrm{~s}$ ). The modulation of the $\Delta \mathrm{T}$ curve between these points is in fact a result of the vacillation phenomenon, not of the control system. A minimum $\Delta \mathrm{T}$ is measured during each wave maximum and vice versa. The LAV is the only flow where such a modulation was measurable (due to the finite conductivity of the walls and the finite residence time of coolant in contact with the sidewalls). It indicates that the total heat transport in the flow is significantly modulated during LAV.

Although waves 2 and 4 are drifting waves, it is worth noting that at high $\Theta$ a stationary wave- 2 component precedes the growth of the drifting waves, i.e. during the LAV minima. The amplitude of the stationary wave- 2 is substantial over this range of $\Delta \mathrm{T}$ and in fact 
sometimes dominates over all other waves when it appears. Other apparently important wavecomponents include a stationary wave- 1 and drifting waves- $3,6,7$ and 8 . The amplitude of the topographically forced wave- 3 component never exceeds $0.8 \mathrm{~mm} / \mathrm{s}$ and can only be said to play a dominating role during brief intervals of wave- 3 amplitude vacillation periods before a wave- 4 growth. A drifting wave- 6 or 8 , on the other hand, sometimes grows to amplitudes almost as large as wave- 4 during the LAV in B9, but the wave- 4 and wave- 2 components always dominate over wave- 6 and 8 , except occasionally for a few moments between the wave4 and wave- 2 peaks. This indicates a complex scenario, possibly of wave-wave interactions, which may have several underlying processes associated with it. Figure 12 illustrates a wave number spectrum time-series, showing that the LAV in B9r, unlike the flows discussed so far, experiences significant amplitude growth and decay during each vacillation cycle over almost the entire measurable wave number spectrum.

Another feature of figure 12 worth noting is the decreasing frequency of the LAV with increasing $\Theta$. In fact the quasi-symmetric flow observed at higher $\Theta$ s could be interpreted as an infinitely long lasting high-amplitude zonal flow phase of the LAV, at least conceptually. The transition between LAV and symmetric flow was located during the reverse scan B9i (at values of $\Theta$ just above those where B9r was initiated). The period of LAV during this step-wise scan increased with $\Theta$, but at a critical value of $\Theta$, LAV ceased to develop.

\subsubsection{Chains of nonlinear wave-wave interactions}

In accordance with the features noted in the general regime diagram (figure 7 of Paper I), wave- 2 becomes an increasingly prominent feature in the LAV regime with increasing $\mathcal{T}$. The presence of wave-2 (like wave-4) is usually pronounced at small radii and an increase in wave- 6 activity is also seen. The relatively strong wave- 4 growth at small radii suggests that a wave-wave interaction between waves 2,4 and 6 might be active, e.g. $4 \rightarrow 2+6$. With the presence of a strong wave- 8 component and the occurence of a significant stationary wave2 and wave- 1 at high $\mathcal{T}$ and $\Theta$, many more wave-wave interactions could be thought of as contributing to the increasingly complex LAV flows. In order to demonstrate the complexity and identify some of the most dominant triad interactions, the time interval corresponding to the fourth wave peak in figure $11 \mathrm{~b}$ has been re-tracked and reanalysed, this time with velocity field measurements every 3 seconds. The results are summarised in figure 13 .

The relatively fast decrease and gradual recovery of the mean flow is shown in figure 13a. Such behavior is reminiscent of a relaxation oscillation (e.g. Namias 1950, Ghil and Childress 1987), which is plausible if there is a competition between baroclinic instability releasing potential energy and diabatic forcing tending to restore it. A few spurious departures from the curve before and after the minimum are due to intractable particle tracking problems resulting in very low resolution velocity fields at these points. Superimposed on the curve is a small high-frequency variability with a time-scale of the order of $\sim 20$ s. The wave-amplitudes for waves 1-9 are shown in figure 13b,c,d in order of decreasing magnitude. Notice the different y-axis scales. The dominant waves are characterised by a double-peak in the amplitude of wave- 4 , followed by a wave- 6 peak and another wave- 4 peak, before wave- 2 dominates the flow until all wave activity has decayed. The second wave- 4 peak coincides with the mean flow minimum. Just prior to this, a strong wave- 5 peak is followed immediately by a clear wave- 9 peak. It is also worth noting the amplitude of wave-1, which is relatively high prior to the LAV compared with the other waves, and it occasionally oscillates around this value during the periods of high wave-activity.

The purpose of figures $13 \mathrm{e}-\mathrm{j}$ is to look for evidence that the resonance conditions for certain triad interactions are satisfied. It tests whether (a) the phases of the waves are locked, i.e. whether their frequencies $\omega(m)$ sum to zero and (b) whether the radial wave-numbers also sum to zero. The latter criterion is tested by distinguishing between whether the absolute phase difference $\Delta \phi$ between the $1 / 4$ and $3 / 4$ gap width is above or below $\pi / 2$, implying a 


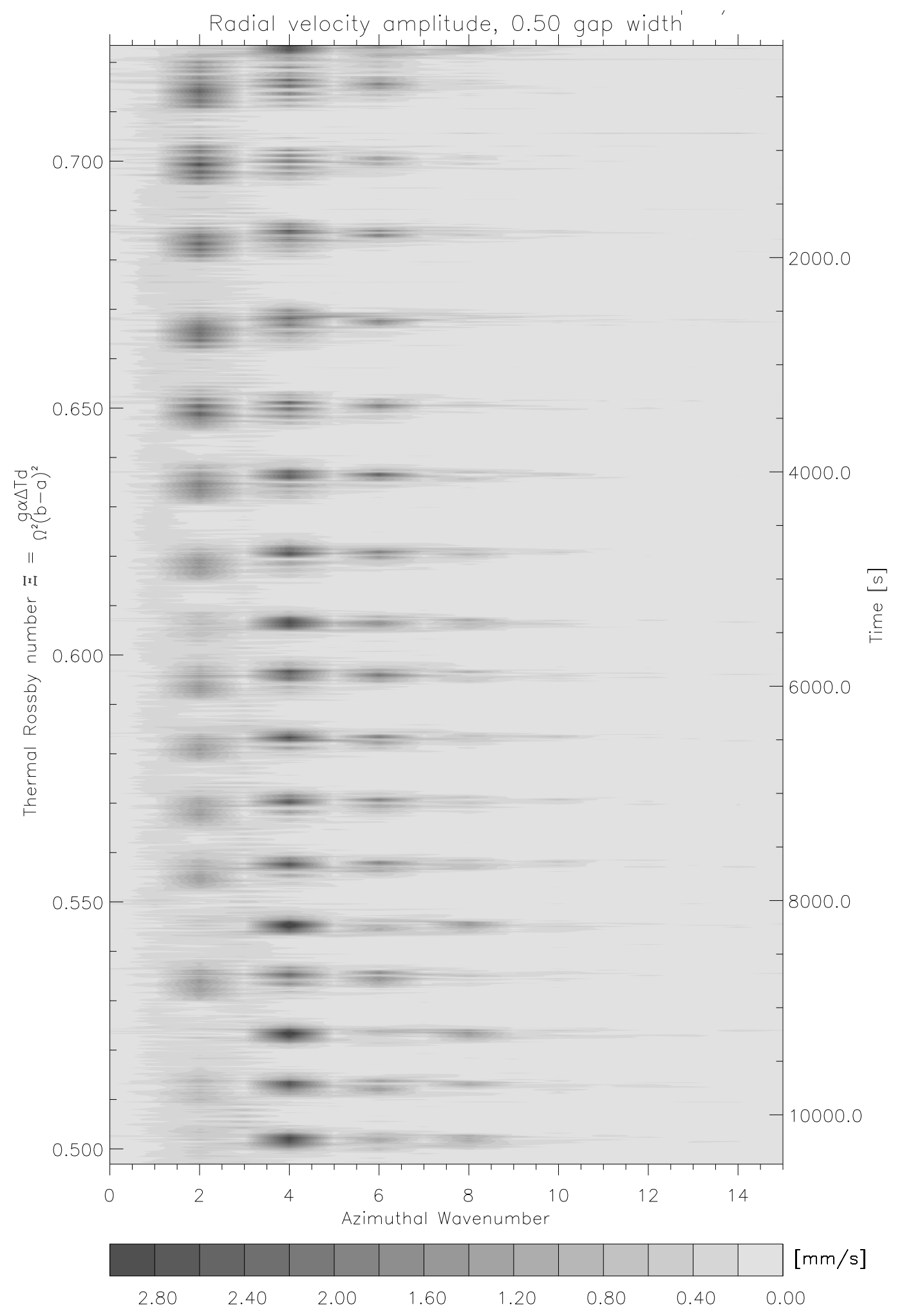

Figure 12. Wave number spectrum time-series at level 1 mid-radius for $\mathrm{B} 9 \mathrm{r}\left(\mathcal{T}=10.71 \times 10^{6}=\right.$ const. $)$

radial wave- 2 or wave- 1 component, respectively. Although this is a rather rough measure it turns out that the 3 wave-components of a triad only rarely meet criterion (b) under this condition for the same period of time when criterion (a) is satisfied. Reference may also be 
made to figure 10 which shows that this simple analysis would identify a $(3,1)-(4,1)-(7,2)$ triad correctly for experiment D1i.

The examples shown in figures 13e-j examine the triads

$$
\begin{aligned}
& (3,1) \leftrightharpoons(4,1) \leftrightharpoons(7,2), \\
& (5,1) \leftrightharpoons(4,1) \leftrightharpoons(9,2) \text { and } \\
& (6,1) \leftrightharpoons(2,1) \leftrightharpoons(8,2),
\end{aligned}
$$

where the phase-locking for the 3-4-7 triad is determined by the difference in frequencies of waves 4 and 7 , while also assuming that a stationary wave- 3 component with zero frequency is present (the phase of wave- 3 turns out to be mainly stationary for the first part of the LAV cycle up to 2500 s before a drifting component temporarily dominates up to about 2750s).

The analysis above suggests that the wave-3-4-7 resonance seems to be associated with the initial wave- 4 growth up to the first peak. The entire duration of the LAV after that seems to be associated with a large variety of nonlinear mechanisms which keep the waveamplitudes high and the mean zonal flow low. The 5-4-9 and the 6-2-8 triads are only two examples of what appears to be a chain of nonlinear interactions. The simple analysis applied above is able to identify triads throughout the cycle. In some cases the appearance of an energetic wave component is preceded by a triad which transfers energy to this wave, e.g. 41-5 (not shown) follows the 3-4-7 resonance and precedes the 5-4-9 interaction. At least part of the wave-5 amplification, however, may also be linked to a 3-2-5 triad which fulfils the resonance conditions for a very brief time during the wave- 5 amplitude growth (by this time the wave- 3 phase was dominanted by a drifting component). The phases of several waves of adjacent wave-number often lock, implying that an interaction via the long wave-1, which remains quasi-stationary, might also play an important role. Generally, however, no systematic evolution from one interaction to another could be found.

Although the analysis here is very simplistic and does not provide an explanation for all aspects of the flow, it demonstrates that (a) the wave-3-4-7 triad plays a crucial role, at least during the initial wave-growth phase of the LAV, (b) the following evolution of the complex LAV is greatly influenced by nonlinear wave-wave interactions and (c) that high wave-numbers, and possibly also the quasi-stationary wave-1, play an important role at certain intervals. A numerical simulation of LAV would ideally be required to perform an in-depth analysis of the wave-energy distribution and evolution.

\section{Discussion}

This study has undertaken an experimental investigation of rotating flows which are subject to both baroclinic and topographically forced waves and instabilities. Our laboratory experiments with the differentially heated rotating annulus with azimuthally varying sinusoidal wave-3 bottom topography have been performed over a wide range of previously sparsely explored parameter space. A rich and complex variety of flows, including some new types of flow regime, were discovered and analysed quantitatively through particle tracking techniques in parallel with high resolution qualitative flow visualisations. A detailed regime diagram was constructed in Paper I which summarised the extent of influence of the observed flows in a region of nondimensional $(\mathcal{T}, \Theta)$ parameter space, where it was found that regimes of symmetric, drifting and stationary wave flows were overlapping.

Within the baroclinic wave regime two distinct time-dependent sub-regimes were identified. These were characterised by low-frequency oscillations which were robust and reproducible. 
One of the sub-regimes was defined here as a stationary wave amplitude vacillation (SAV), which was characterised by an oscillation period of around 30-60 annulus revolutions. The other sub-regime was defined as low-frequency amplitude vacillation (LAV), and differed significantly from any annulus flow regimes reported in the present or previous studies. The period of oscillation for LAV was very long and corresponded to around 100-180 annulus revolutions.

A common thread through both of these time-dependent regimes was that both seem to involve nonlinear wave-wave and wave-zonal flow interactions in the form of one or more resonant wave triads. Some of the most important of these include components that are forced directly by the zonally-varying topography. Such a notion has a long history dating back to the suggestion by Egger (1978) that an interaction of a topographically forced wave with two traveling Rossby waves play an important role in explaining the persistence of blocking events (see also Ghil and Childress 1987, Section 6.2). More recent work has invoked similar stationary-traveling wave interactions to account for a variety of large-scale dynamical phenomena, including intraseasonal oscillations in the Earth's extratropics (Jin and Ghil 1990, Dickey et al. 1991, Koo and Ghil 2002) and stratospheric sudden warmings Andrews et al. (1987), O'Neill and Pope (1988), Charlton and Polvani (2007), Charlton et al. (2007), Matthewman et al. (2009) as well as various phenomena observed in laboratory experiments (e.g. Buzyna et al. 1989, Ohlsen and Hart 1989, Pfeffer et al. 1990).

Although some reasonably clear results have emerged from our experiments with some identifiable flow regimes with clearly delineated properties, some caution may be needed over our use of continuously or stepwise swept control parameters. Such parametric variations are well known to be capable of inducing additional nonlinear phenomena and/or leading to quantitative shifts in the location of bifurcations (e.g. Bishop and Galvanetto 1993, Hua and Lu 2001, Kogan 2007), especially if such variations are non-monotonic or even cyclic in nature (e.g. Buzyna et al. 1978, Castrejón-Pita and Read 2010). We have tried to be careful to vary our parameters sufficiently slowly to avoid the parameter sweeps having too much influence on the phenomena observed. Nevertheless, we cannot rule out the possibility that time-varying parameters have affected the form and location of some of the flow transitions we have observed. This should perhaps be a significant focus for future work.

\subsection{Stationary Amplitude Vacillation - period $\sim$ 30-60 revolutions}

It was not immediately obvious from the streak movie observations that the drifting wave-3 component might play a crucial role in SAV. It was shown by careful quantitative analysis of the regime scans that the mechanism underlying the SAV flow involved the interaction of a stationary and a drifting component of wave-3, in which the time-scale of one AV cycle also corresponded to the drift period of the drifting wave-3. The SAV variability characteristics, including the period of one cycle of about 30-60 annulus revolutions, thus appeared to be fundamentally similar to those observed in a previous study by Bernardet et al. (1990) and Pfeffer et al. (1990).

It was also possible in the present experiments to determine the extent in nondimensional parameter space of the SAV mode of variability. This was defined by the boundaries of the overlapping drifting and stationary wave regimes (both found to be dominated by wave-3 in SAV). This clearly separated the SAV regime from other low-frequency variability regimes, including the LAV regime discussed in Section 4 and below, and possibly another regime at very low $\Theta$ and high $\mathcal{T}$. The latter also involved the growth and decay of a quasi-stationary wave-3 in an otherwise steady wave-5 flow, but (judging from just a single qualitative streak movie taken during experiment A4i) it was characterised by a comparatively more irregular and lower frequency variability than the SAV. Another result from the present investigation, which is of interest for comparison with barotropic annulus experiments (e.g. Weeks et al. 1997, 
Tian et al. 2001), is that SAV does not simply involve intermittent irregular switching between a stationary wave-3 and a drifting wave-3. Such a scenario would resemble conceptually the temporal behaviour observed in the barotropic annulus experiments of Weeks et al. (1997) and Tian et al. (2001) over a region of parameter space where two regimes, namely the blocked and unblocked flows, overlap. In the SAV flow regime, however, there was an underlying temporal periodicity. There is also a region of parameter space between the SAV and the stationary wave regime (see Fig. 7 of Paper I), which has a distinctly different character, namely a quasi-steady drifting wave- 4 .

The identification of similarities between the present experiments and those of Bernardet et al. (1990) is of some interest since, in Bernadet et al.'s case, the forced stationary wave was wavenumber 2 while the vacillating drifting wave was wave- 6 . There was a clear scale separation, therefore, between the two interacting components, which has been suggested by Jin and Ghil (1990) as being of some fundamental significance to low-frequency variability phenomena in the atmosphere. For the study of the Earth's tropospheric dynamics this may indeed represent an interesting analogy, since there is typically a scale separation in the atmosphere between the transient baroclinic waves (wavenumbers 6-9) and the stationary waves (1-3). The present experiments have demonstrated, however, that such a separation of scales is not fundamentally essential for low-frequency variability to occur on time-scales of 30-60 annulus revolutions. This may be of some interest also for the mid-latitude atmospheric dynamics on Mars (see e.g. Hollingsworth et al. 1996) where the horizontal scales of the baroclinic weather systems are more comparable to the scale of the underlying topography.

\subsection{Low frequency Amplitude Vacillation - period $\sim$ 100-180 revolutions}

The LAV flow regime, as observed in our experiments, was primarily characterised by the quasi-periodic or irregular growth and decay of drifting waves, of which wave-4 usually dominated. Simpler examples of LAV were distinguished from more complicated forms in terms of Fourier wave-spectra and time-series. These more complex LAV flows tended to occur at larger values of $\mathcal{T}$, suggestive of a gradual transition towards ever more "turbulent" behavior as the effects of viscous dissipation are reduced. In such cases, time series of wave amplitude and the strength of the mean zonal flow showed that a large number of distinct wave modes grew to substantial amplitudes during a complex LAV, while significantly fewer modes were involved in the simpler LAV. The mechanism behind the simpler LAV vacillation cycle was found to involve nonlinear interactions with the topographically forced wave, whereby a resonant triad enabled the transfer of energy from the forced wave-3 to wave numbers 4 and 7 which had approximately equal drift frequencies. At the same time a significant weakening of the vertical shear of the mean zonal flow was observed, resulting in a sharp decrease in the strength of the mean flow near the top boundary and an increase near the bottom. A mechanism for a repetition of the cycle was proposed whereby the increased mean flow near the bottom would cause the forced wave- 3 to drift by one wavelength, thereby temporarily causing the $m=3-4-7$ triad to violate the necessary conditions for resonance and allowing the mean flow structure to re-establish a strong vertical shear. The $m=3-4-7$ triad also played a role in the initial wave growth phase of the more complex LAV.

\subsection{LAV and stratospheric SSWs?}

It is suggested that a possible geophysical analogy to LAV may be found in the winter midlatitude phenomenon of stratospheric sudden warmings (SSW). A comparison between a typical annulus experiment and the general circulation of a planetary atmosphere usually assumes that one full $360^{\circ}$ revolution of the annulus (relative to the stationary laboratory) corresponds 
to 1 'day'. The fact that major sudden stratospheric warmings usually occur only once per winter, and then mostly towards the end of that season, already suggests that they may be the result of an internal dynamical mechanism which has a time-scale of a few months, ie $\geq 100$ days. If the typical winter conditions favouring an eastward zonal jet were to persist for longer than one season, the possibility arises that SSWs might occur repeatedly with cycles of similar periods. Indeed, numerical studies with persistent winter solstice conditions have been carried out recently (e.g. by Yoden et al. 1999 and Gregory 1999), which simulate cyclically recurring (though generally aperiodic) minor or major SSW events. However, suggestions as to what the underlying mechanism or cause of the event may be are still controversial.

In order to illustrate the potential of the present experiments as a vehicle to simulate and study flows analogous to SSW events, figure 14 compares the much-studied major warming event of 1979 to the complex LAV cycle analysed in figure 13. The time-series in figure 14 shows the amplitude of the mean flow and the two most dominant wave-modes at the moderately high level in the atmosphere of $\sim 10 \mathrm{hPa}$, while figure 13 is located at the top level $1(z=12.4$ $\mathrm{cm})$ in the annulus experiments. In the case of the 1979 warming, the wave amplitudes are given in terms of geopotential height and have wave-numbers 1 and 2, while the behaviour of the dominant waves 4 and 2 for the laboratory LAV are shown in terms of radial velocity amplitude.

Perhaps the most striking similarity is the time-scale (measured in 'days') over which both events occur. Robust features in both time-series include

(a) the relatively poleward or radially inward position of the zonal mean jet maximum (figure $14 \mathrm{a}, \mathrm{d})$,

(b) the (fluctuating) growth of the dominating wave towards a maximum amplitude which coincides with the mean flow minimum, followed by a (fluctuating) decay back to very small amplitude (figure $14 \mathrm{c}, \mathrm{f}$ ),

(c) the delayed growth to a slightly lower amplitude maximum of wave- 2 as well as an amplitude vacillation at low amplitudes prior to the main event, which is roughly in anti-phase with the fluctuations in the mean flow (figure $14 \mathrm{~b}, \mathrm{e}$ ).

Such direct comparisons, of course, have their limitations, and it is not the aim here to precisely simulate with a laboratory model specific events in the terrestrial atmosphere. Since there is no seasonal cycle in the present annulus experiments, the comparison in figure 14 necessarily breaks down at the end of winter where, in the stratosphere, weak summer easterlies are gradually expected to form, while in the annulus experiments the eastward jet grows back to its 'normal' large amplitude. A sudden reversal to easterlies which occurs, by definition, in the 'major' warming of 1979 down to altitudes of $\sim 10 \mathrm{hPa}$, has not been observed in any LAV events in the present experiments. This does not rule out the possibility, however, that such a reversal might be seen in the annulus experiments as part of an internal dynamical LAV mechanism which might involve interactions with other dominant wave-numbers. In this respect it is worth pointing out that an upper easterly and lower westerly flow has been observed in the LAV regime in the presence of a quasi-steady drifting wave-3. Although this flow did not involve low-frequency variability, it demonstrates the possibility of multiple solutions associated with very distinct vertical mean flow structures in the LAV region of parameter space.

In this respect the experiments may shed some light onto a more general, but equally important and topical, issue concerning the interannual variability of SSWs (e.g. Butchart et al. 2000, Limpasuvan et al. 2004, Charlton and Polvani 2007, Black and McDaniel 2007, Sun et al. 2012)), and in particular the observation that major SSWs do not occur every winter. There have been suggestions that the (sometimes) quasi-biennial occurrence of major SSWs may be linked to the quasi-biennial oscillation in the tropical stratosphere (Holton and Tan 1980). However, there also exist many observations where major SSWs have not 
occurred for several years in a row, e.g. between 1992 and 1998. A fundamental question arises, therefore, as to what the underlying reasons for this variability might be. In particular it is of fundamental interest whether this variability is intrinsic to the internal dynamics of the stratosphere or whether a variation of external conditions is the main cause, or a combination of both. For the purpose of this discussion, the rotating annulus in its present configuration may be understood as representing a highly simplified model of a mid-latitude atmosphere.

Clearly there are no 'extrinsic' processess in the rotating annulus corresponding to tropical ocean SSTs, high latitude dynamical influences or chemical effects. The analogy suggested here between SSWs and LAV would therefore imply that the dynamical evolution of a SSW could be purely intrinsic to the mid-latitude stratosphere (including the forcing region of the troposphere). One could then argue further that the stratosphere should also be able to support stable flows which differ from those involving SSWs under the same external parameter conditions, by analogy with the multiple stable states discovered in the same region of parameter space where LAV occurred (including a weak, steady wave- 4 flow or a drifting wave-3 with an upper easterly jet - see Paper I). The actual flow developing in winter would thus be sensitive to the initial conditions. Since the internal conditions in a complex system like the atmosphere are never exactly the same every year, the laboratory evidence supporting the notion of multiple equilibria may provide some insight into the interannual variability of major SSWs.

In this regard, annulus experiments of the kind reported here may provide additional insights e.g. into the possible role of the seasonal cycle in inducing complex long-term variability in systems supporting coexisting almost intransitive states, along the lines suggested by various authors (e.g. Buzyna et al. 1978, Lorenz 1990, Itoh and Kimoto 1996, Ghil and Robertson 2002). These studies showed that slow and irregular variability is often associated with various forms of "chaotic itinerancy" as a system wanders among a finite set of almost intransitive states. Slow, season-like cyclic modulations in regions of parameter space close to where flow transitions occur (e.g. between different wavenumber states), in particular, can lead to slow, chaotic variability which more recent work (e.g. Castrejón-Pita and Read 2010) has shown can even lead to periodic or chaotic states that are synchronized with the forcing.

With regard to SSWs, the mismatch between the wavenumbers of the dominating wave modes in the LAV and SSW events inevitably complicates a closer examination of dynamical similarities or analogies. Another point of disagreement between observed SSWs and the laboratory LAV was in the phase drift of the dominant wave in the LAV regime. In most of the SSWs in the northern hemisphere the wave-1 is quasi-stationary during its peak amplitude, following a slow eastward drift of a fraction of a wave-length which is sometimes even preceded by a short westward drift. Wave- 4 during LAV in the present annulus experiments, on the other hand, was observed to drift by several wave-lengths during the cycle with the slowest drift speeds at its largest amplitude. In light of these results, however, it will be of interest to determine from further numerical model experiments in the stratosphere (e.g. with constant seasonal conditions) whether SSWs arise in principle as a sustained cyclic internal mode of oscillation or via some other process.

\section{Acknowledgements}

The laboratory work has greatly benefitted from the help of Dr. D. Smith of the Oxford University Physics Electronics Services Group, to whom we would like to extend special thanks, in particular for installing the new hardware components in the upgraded annulus system. SHR was part-funded in this work by a research studentship from the UK Natural Environment Research Council. We are grateful to an anonymous referee and Prof. Michael Ghil for their comments on an earlier version of this manuscript which significantly enhanced the discussion 
and presentation.

\section{References}

Anderson, J. and Rosen, R., The latitude-height structure of 40-50 day variations in atmospheric angular momentum. J. Atmos. Sci., 1983, 40, 1584-1591.

Andrews, D., Holton, J. and Leovy, C., Middle Atmosphere Dynamics, 1987 (Academic Press, INC.).

Barnes, J. and Hollingsworth, J., Dynamical modeling of a planetary wave mechanism for a Martian polar warming. Icarus, 1987, 71, 313-334.

Bernardet, P., Butet, A., Déqué, M., Ghil, M. and Pfeffer, R., Low-frequency oscillations in a rotating annulus with topography. J. Atmos. Sci., 1990, 47, 3023-3043.

Birner, T. and Williams, P.D., Sudden Stratospheric Warmings as Noise-Induced Transitions. J. Atmos. Sci., 2008, 65, 3337-3343.

Bishop, S.R. and Galvanetto, U., The behaviour of nonlinear oscillators subjected to ramped forcing. Meccanica, 1993, 28, 249-256.

Black, R.X. and McDaniel, B.A., The Dynamics of Northern Hemisphere Stratospheric Final Warming Events. J. Atmos. Sci., 2007, 64, 2932-2946.

Branstator, G.W., A striking example of the atmosphere's leading traveling pattern. J. Atmos. Sci., 1987, 44, 2310-2323.

Butchart, N., Austin, J., Knight, J., Scaife, A. and Gallani, M., The response of the stratospheric climate to projected changes in the concentrations of well-mixed greenhouse-gas from 1992 to 2051. J. Clim., 2000, 13, $2142-2159$.

Buzyna, G., Pfeffer, R.L. and Kung, R., Cyclic variations of the imposed temperature contrast in a thermally driven annulus of fluid. J. Atmos. Sci., 1978, 35, 859-881.

Buzyna, G., Pfeffer, R.L. and Kung, R., Kinematic properties of wave amplitude vacillation in a thermally driven rotating fluid. J. Atmos. Sci., 1989, 46, 2716-2729.

Castrejón-Pita, A. and Read, P.L., Synchronization in a Pair of Thermally Coupled Rotating Baroclinic Annuli: Understanding Atmospheric Teleconnections in the Laboratory. Phys. Rev. Lett., 2010, 104, 204501.

Charlton, A.J. and Polvani, L.M., A New Look at Stratospheric Sudden Warmings. Part I: Climatology and Modeling Benchmarks. J. Clim., 2007, 20, 449-469.

Charlton, A.J., Polvani, L.M., Perlwitz, J., Sassi, F., Manzini, E., Shibata, K., Pawson, S., Nielsen, J.E. and Rind, D., A New Look at Stratospheric Sudden Warmings. Part II: Evaluation of Numerical Model Simulations. J. Clim., 2007, 20, 470-488.

Dickey, J.O., Ghil, M. and Marcus, S.L., Extratropical aspects of the 40-50 day oscillation in length of day and atmospheric angular momentum. J. Geophys. Res., 1991, 96, 22643-22658.

Egger, J., Dynamics of blocking highs. J. Atmos. Sci., 1978, 35, 1788-1801.

Esler, J.G. and Scott, R.K., Excitation of transient Rossby waves on the stratospheric polar vortex and the barotropic sudden warming. J. Atmos. Sci., 2005, 62, 3661-3682.

Fjørtoft, R., On changes in the spectral distribution of kinetic energy in two-dimensional, non-divergent flow. Tellus, 1953, 5, 225-230.

Früh, W. and Read, P., Wave interactions and the transition to chaos of baroclinic waves in a thermally driven rotating annulus. Phil. Trans. R. Soc. Lond. A, 1997, 355, 101-153.

Ghil, M., Dynamics, statistics and predictability of planetary flow regimes. In lrreversible Phenomena and Dynamical Systems Analysis in the Geosciences, edited by C. Nicolis and G. Nicolis, pp. 241-283, 1987 (D. Reidel: Dordrecht/Boston/Lancaster).

Ghil, M. and Childress, S., Topics in Geophysical Fluid Dynamics: Atmospheric Dynamics, Dynamo Theory and Climate Dynamics, 1987 (Springer-Verlag).

Ghil, M. and Robertson, A.W., "Waves" vs. "particles" in the atmosphere's phase space: a pathway to longrange forecasting?. PNAS, 2002, 99, 2493-2500.

Gregory, A., Numerical experiments in winter stratospheric dynamics. Ph.D. Thesis, Univ. Reading, 1999.

Hignett, P., Characteristics of amplitude vacillation in a differentially heated rotating fluid annulus. Geophys. Astrophys. Fluid Dyn., 1985, 31, 247-281.

Hignett, P., White, A., Carter, R., Jackson, W. and Small, R., A comparison of laboratory measurements and numerical simulations of baroclinic wave flow in a rotating cylindrical annulus. Quart. J. R. Met. Soc., 1985, 111, 131-154.

Hollingsworth, J., Haberle, R., Barnes, J., Bridger, A., Pollack, J., Lee, H. and Schaeffer, J., Orographic control of storm zones on Mars. Nature, 1996, 380, 413-416.

Holton, J. and Tan, H., The influence of the equatorial quasi-biennial oscillation on the global circulation at 50 mb. J. Atmos. Sci., 1980, 37, 2200-2208.

Hua, C.C. and Lu, Q.S., Time-Dependent Bifurcation: A New Method and Applications. Int. J. Bifurcation Chaos Appl. Sci. Eng., 2001, 11, 3153-3162.

Itoh, H. and Kimoto, M., Multiple attractors and chaotic itinerancy in a quasi-geostrophic model with realistic topography: implications for weather regimes and low frequency variability. J. Atmos. Sci., 1996, 53, 22172231. 
James, I. and James, P., Ultra-low frequency variability in a simple atmospheric circulation model. Nature, 1989, 342, 53-55.

James, I. and James, P., Spatial structure of ultra-low frequency variability of the flow in a simple atmospheric circulation model. Quart. J. R. Met. Soc., 1992, 118, 1211-1233.

James, P., Fraedrich, K. and James, I., Wave-zonal flow interaction and ultra-low frequency variability in a simplified global circulation model. Quart. J. R. Met. Soc., 1994, 120, 1045-1067.

Jin, F.F. and Ghil, M., Intraseasonal oscillations in the extratropics: Hopf bifurcation and topographic instabilities. J. Atmos. Sci., 1990, 47, 3007-3022.

Jonas, P., Laboratory observations of the effects of topography on baroclinic instability. Quart. J. R. Met. Soc., 1981, 107, 775-792.

Kogan, O., Controlling transitions in a Duffing oscillator by sweeping parameters in time. Phys. Rev. E, 2007, 76, 037203 .

Koo, S. and Ghil, M., Successive bifurcations in a simple model of atmospheric zonal-flow vacillation. Chaos, 2002, 12, 300-309.

Kuroda, T., Medvedev, A.S., Hartogh, P. and Takahashi, M., On Forcing the Winter Polar Warmings in the Martian Middle Atmosphere during Dust Storms. J. Met. Soc. Jpn., 2009, 87, 913-921.

Kushnir, Y., Retrograding wintertime low-frequency disturbances over the North Pacific Ocean. J. Atmos. Sci., 1987, 44, 2727-2742.

Labitzke, K., The amplification of height Wave 1 in January 1979: a characteristic precondition for the major warming in February. Mon. Wea. Rev., 1981, 109, 983-989.

Leach, H., Thermal convection in a rotating annulus: Effects due to bottom topography. J. Fluid Mech., 1981, 109, $75-87$.

Li, G., Kung, R. and Pfeffer, R., An experimental study of baroclinic flows with and without two-wave bottom topography. J. Atmos. Sci., 1986, 43, 2585-2599.

Limpasuvan, V., Thompson, D.W.J. and Hartmann, D.L., The Life Cycle of the Northern Hemisphere Sudden Stratospheric Warmings. J. Clim., 2004, 17, 2584-2596.

Lorenz, E., Can chaos and intransitivity lead to internal variability?. Tellus, 1990, 42A, 378-389.

Marshall, S.D. and Read, P.L., An experimental investigation into topographic resonance in a baroclinic, rotating annulus. Geophys. Astrophys. Fluid Dyn., 2015, p. submitted.

Matsuno, T., A dynamical model of the stratospheric sudden warming. J. Atmos. Sci., 1971, 28, 1479-1494.

Matthewman, N.J., Esler, J.G., Charlton-Perez, A.J. and Polvani, L.M., A New Look at Stratospheric Sudden Warmings. Part III: Polar Vortex Evolution and Vertical Structure. J. Clim., 2009, 22, 1566-1585.

McEwan, A., Mander, D. and Smith, R., Forced resonant second-order interaction between damped internal waves. J. Fluid Mech., 1972, 55, 589-608.

Namias, J., The index cycle and its role in the general circulation. J. Met., 1950, 7, 130-139.

Ohlsen, D.R. and Hart, J.E., Nonlinear interference vacillation. Geophys. Astrophys. Fluid Dyn., 1989, 45, $213-235$.

O'Neill, A. and Pope, V., Simulations of linear and nonlinear disturbances in the stratosphere. Quart. J. R. Met. Soc., 1988, 114, 1063-1110.

Pfeffer, R., Ahlquist, J., Kung, R., Chang, Y. and Li, G., A study of baroclinic wave behaviour over bottom topography using complex principal component analysis of experimental data. J. Atmos. Sci., 1990, 47, $67-81$.

Plumb, R., Instability of the distorted polar night vortex: a theory of stratospheric warmings. J. Atmos. Sci., 1981, 38, 2514-2531.

Read, P., Bell, M., Johnson, D. and Small, R., Quasi-periodic and chaotic flow regimes in a thermally driven, rotating fluid annulus. J. Fluid Mechanics, 1992, 238, 599-632.

Read, P. and Risch, S., A laboratory study of global-scale wave interactions in baroclinic flow with topography I: multiple flow regimes. Geophys. Astrophys. Fluid Dyn., 2011, 105, 128-160.

Scott, R.K., Polvani, L.M. and Waugh, D.W., Internal Variability of the Winter Stratosphere. Part II: TimeDependent Forcing. J. Atmos. Sci., 2008, 65, 2375-2388.

Sun, L., Robinson, W.A. and Chen, G., The Predictability of Stratospheric Warming Events: More from the Troposphere or the Stratosphere?. J. Atmos. Sci., 2012, 69, 768-783.

Tian, Y., Weeks, E.R., Ide, K., Urbach, J.S., Baroud, C.N., Ghil, M. and Swinney, H.L., Experimental and numerical studies of an eastward jet over topography. J. Fluid Mech., 2001, 438, 129-157.

Wallace, J.M., North Atlantic Oscillation/annular mode: two paradigms - one phenomenon. Quart. J. R. Met. Soc., 2000, 126, 791-806.

Weeks, E.R., Tian, Y., Urbach, J.S., Ide, K., Swinney, H.L. and Ghil, M., Transitions between blocked and zonal flows in a rotating annulus with topography. Science, 1997, 278, 1598-1601.

Wilson, R.J., A general circulation model simulation of the Martian polar warming. Geophys. Res. Lett., 1997, 24, 123-126.

Yoden, S., Bifurcation properties of a stratospheric vacillation model. J. Atmos. Sci., 1987, 44, 1723-1733.

Yoden, S., Yamaga, T., Pawson, S. and Langematz, U., A Composite Analysis of the Stratospheric Sudden Warmings Simulated in a Perpetual January Integration of the Berlin TSM GCM. J. Met. Soc. Jpn., 1999, 77, 431-445. 


\section{Appendix A: Experimental parameters} Table A1. Details of the laboratory and numerical experiments. The endings $\mathrm{i}$ and $\mathrm{r}$ indicate the direction of the scan: The
varying parameter is either gradually increased (i) or reduced (r). Only the continuously or frequently sampled light levels are
indicated, occasionally other light levels were viewed. This is Table 3 of Paper I.

\begin{tabular}{|l|c|c|c|c|c|c|}
\hline Exp $^{t}$ & $\mathcal{T}\left[\times 10^{6}\right]$ & \multicolumn{2}{|c|}{ External parameters } & \multicolumn{1}{|c|}{$\begin{array}{c}\text { Duration } \\
{[\text { hight }}\end{array}$} & $\begin{array}{c}\text { Light } \\
\text { level }\end{array}$ \\
\hline A1r & $16.10-0.994$ & $0.131-2.13$ & $2.03-0.759$ & 3.99 & $18: 00$ & 1 \\
A1i & $1.21-16.90$ & $1.76-0.125$ & $0.861-2.08$ & 3.99 & $18: 00$ & 1 \\
A2r & $15.80-5.67$ & $0.264-0.737$ & $2.03-1.22$ & 8.04 & $13: 00$ & 1 \\
A2i & $6.15-36.77$ & $0.679-0.114$ & $1.27-3.10$ & 8.04 & $18: 00$ & 1 \\
A3r & $15.95-0.985$ & $0.066-1.068$ & $2.03-0.505$ & 2.04 & $16: 00$ & 1 \\
A4r & $38.92-4.05$ & $0.086-0.827$ & $3.05-1.01$ & 6.08 & $29: 00$ & 1 \\
A4i & $4.05-61.60$ & $0.827-0.054$ & $1.01-3.91$ & 6.08 & $29: 00$ & 1 \\
B1r & 12.31 & $0.556-0.072$ & 1.80 & $14.47-1.86$ & $3: 30$ & 2 \\
B3r & 20.64 & $0.416-0.023$ & 2.33 & $17.90-0.265$ & $3: 30$ & 2 \\
B6r & $47.91-8.80$ & $0.128-0.702$ & $3.55-1.52$ & 12.68 & $6: 30$ & 2 \\
B6i & $7.12-59.70$ & $0.877-0.102$ & $1.37-3.96$ & 12.69 & $6: 30$ & 2 \\
B7r & $23.53-5.56$ & $0.13-0.566$ & $2.49-1.20$ & 6.47 & $4: 00$ & 2 \\
B7i & $5.41-62.13$ & $0.58-0.050$ & $1.20-4.04$ & 6.44 & $6: 00$ & 2 \\
B9r & 10.71 & $0.70-0.012$ & 1.68 & $16.16-0.26$ & $9: 30$ & 1 \\
B9i & 10.71 & $0.036-0.880$ & 1.68 & $0.65-21.35$ & $10: 20$ & 1 \\
C1r & 6.73 & $0.654-0.039$ & 1.34 & $8.85-0.92$ & $1: 30$ & 2 \\
C1i & 6.73 & $0.139-0.654$ & 1.34 & $0.93-8.80$ & $1: 30$ & 2,4 \\
C2r & 1.85 & $0.465-0.182$ & 0.70 & $1.82-0.70$ & $1: 30$ & 2 \\
C2i & 1.85 & $0.182-0.465$ & 0.70 & $0.71-1.83$ & $1: 30$ & 2 \\
C3r & 1.84 & $2.50-0.577$ & 0.70 & $9.59-2.07$ & $3: 00$ & $1-4$ \\
C3i & 1.84 & $0.498-2.40$ & 0.70 & $1.93-9.50$ & $3: 00$ & 2,4 \\
C4r & 0.671 & $5.44-0.979$ & 0.43 & $7.71-1.29$ & $3: 00$ & $1-4$ \\
C4i & 0.671 & $0.906-5.474$ & 0.43 & $1.20-7.81$ & $3: 00$ & $1-4$ \\
C5r & 8.60 & $0.750-0.172$ & 1.56 & $14.54-3.13$ & $3: 00$ & $1-4$ \\
C5i & 8.60 & $0.179-0.776$ & 1.57 & $3.34-14.91$ & $3: 00$ & 2,4 \\
D1r & $18.05-4.92$ & 0.450 & $2.35-1.23$ & $16.92-5.29$ & $19: 30$ & 2,4 \\
D1i & $0.671-23.75$ & 0.4500 & $0.43-2.53$ & $0.53-21.68$ & $23: 30$ & 2,4 \\
D2r & $40.28-1.02$ & 0.155 & $4.07-0.28$ & $18.44-0.38$ & $17: 00$ & 2,4 \\
D2i & $1.24-26.04$ & 0.155 & $0.61-3.91$ & $0.46-17.51$ & $17: 00$ & 2,4 \\
D3r & $37.77-8.21$ & 0.127 & $3.14-1.47$ & $10.03-2.13$ & $4: 30$ & 2 \\
\hline & & & & & & \\
\hline
\end{tabular}




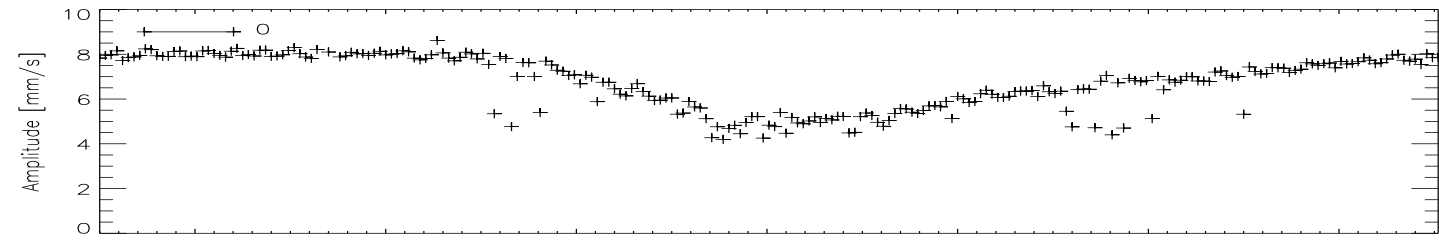

(a)

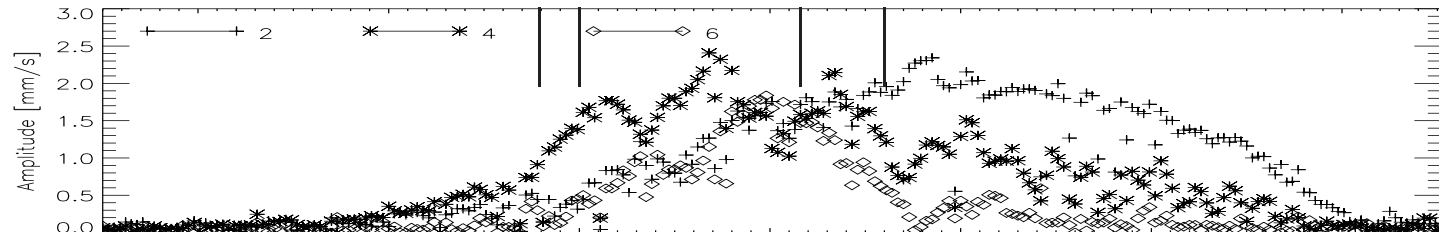

(b)

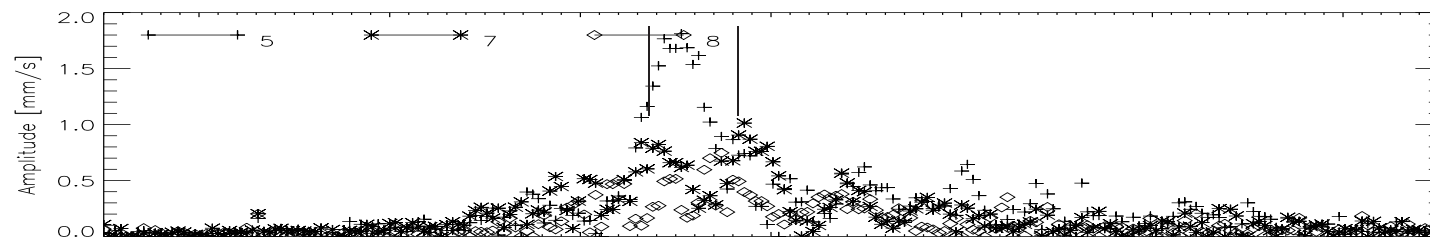

(c)

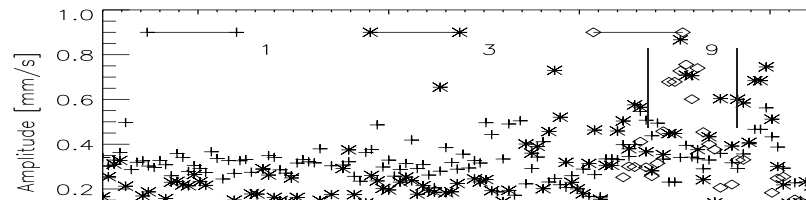

0.0 .

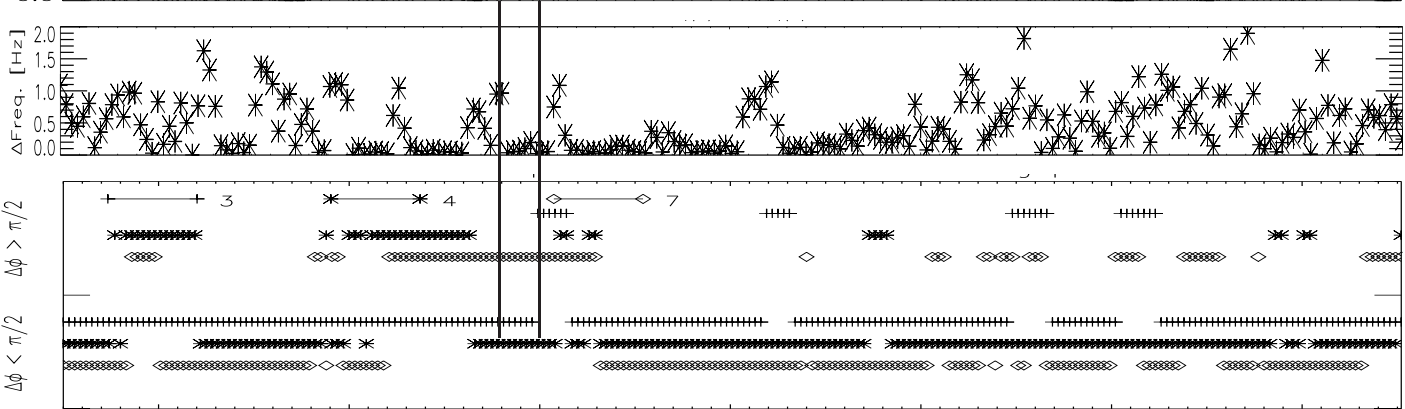

(e)

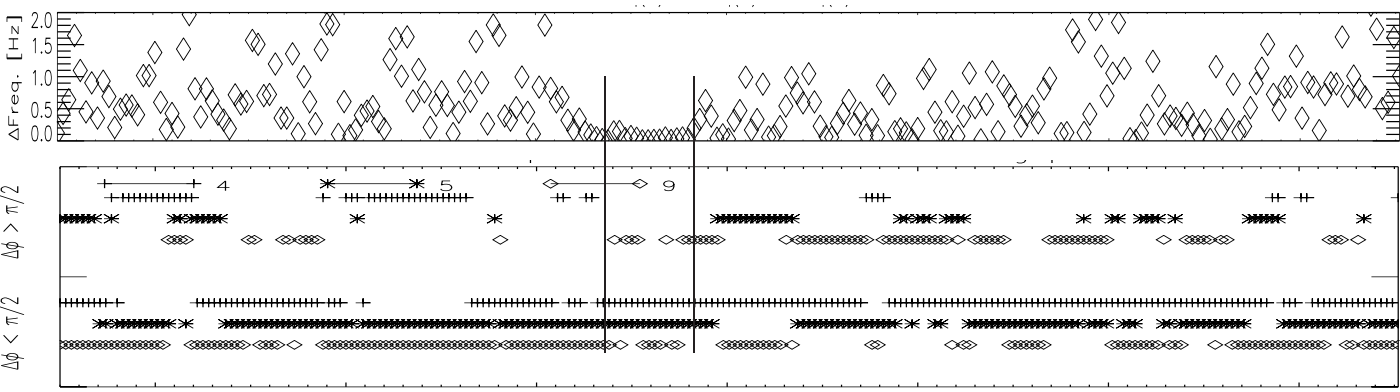

$(\mathrm{g})$

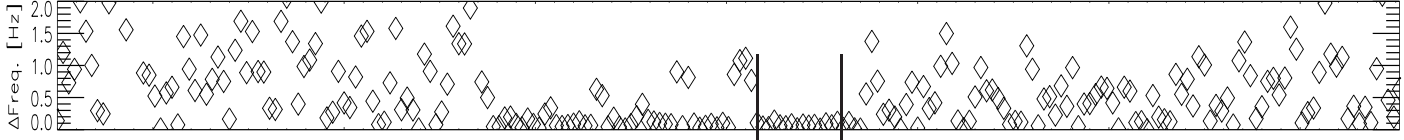

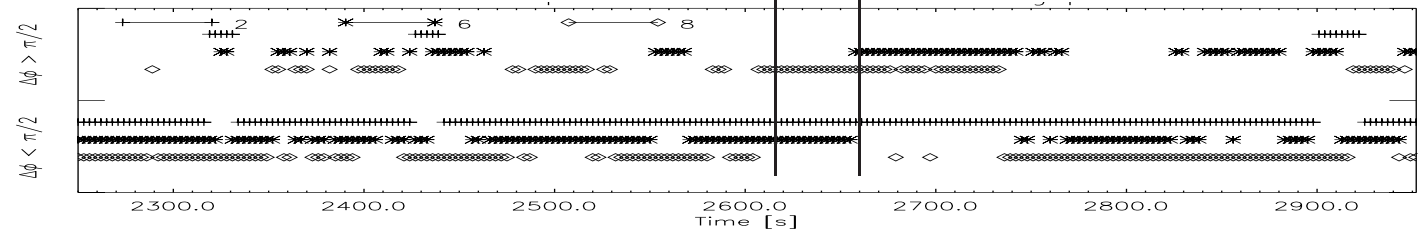

Figure 13. Examples of triad interactions in a complex LAV cycle diagnosed by mean flow (a), wave amplitudes (b,c,d), phase locking (e,g,i) [determined by the difference in frequency $|\Delta \omega|$ between wavenumbers: (e) $|\omega(7)-\omega(4)|,(\mathrm{g}) \mid \omega(9)-$ $\omega(5)-\omega(4) \mid$, (i) $|\omega(8)-\omega(6)-\omega(2)|]$ at mid-radius, and radial wavenumber $(\mathrm{f}, \mathrm{h}, \mathrm{j})$, [indicated by the phase difference $\Delta \phi$ between $r=1 / 4$ and $3 / 4$ gap width], all at level 1 . The vertical lines indicate the time intervals over which a certain wave-triad is likely to play an important role. 
(a)

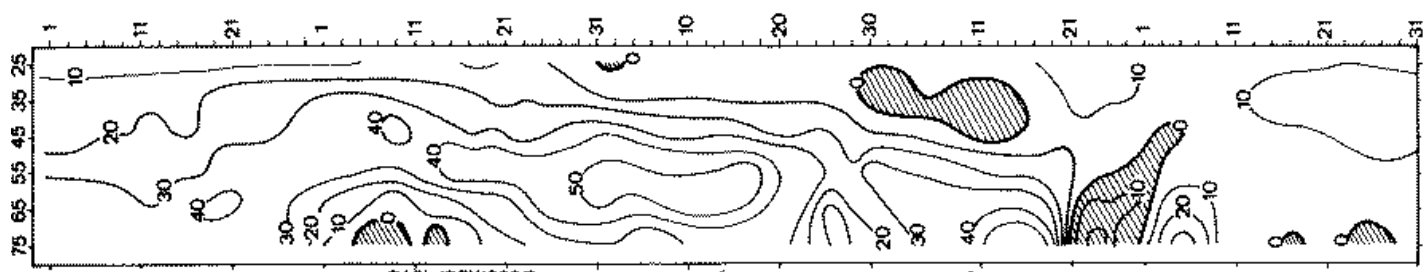

(b)

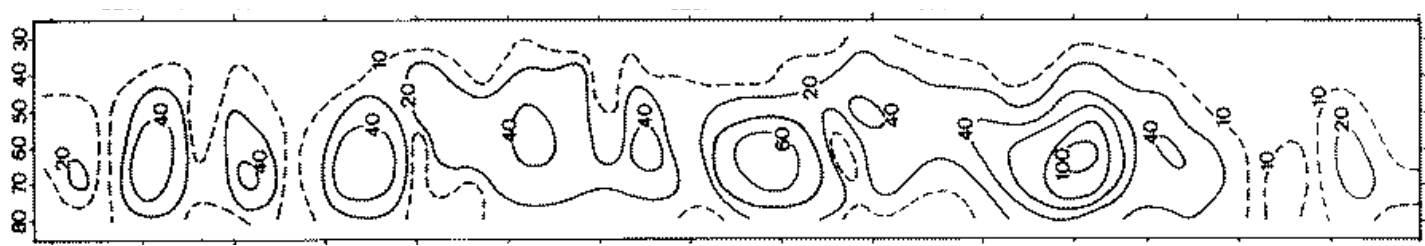

(c)

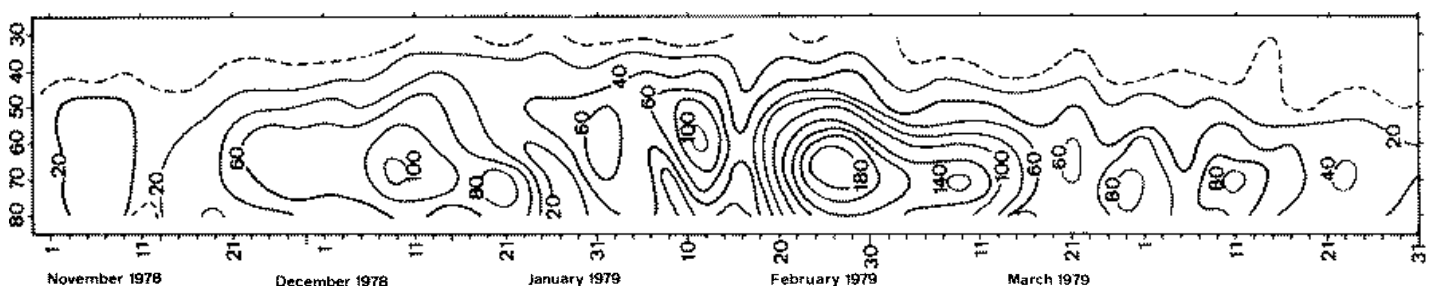

Time [annulus rotations]
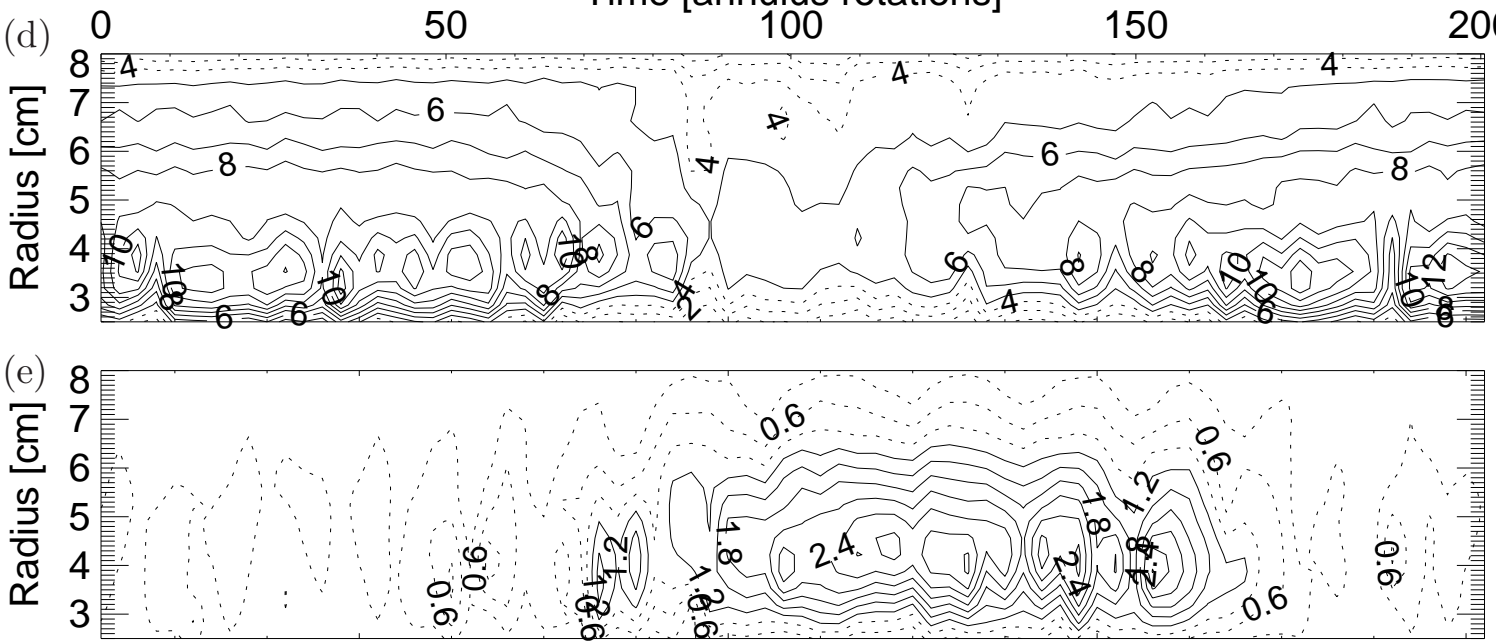

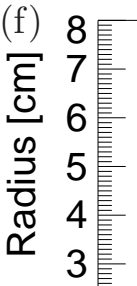

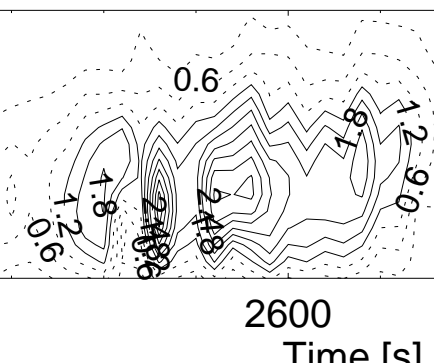

Figure 14. Latitude-time sections at (a,b,c) $10 \mathrm{mb}$ of the stratospheric sudden warming event of 1979 (from Labitzke 1981, Fig. 4, with permission) compared with (d,e,f) radius-time sections at level 1 in experiment B9r; (a) mean zonal wind $\left(\mathrm{m} \mathrm{s}^{-1}\right)$, (b) wave-2 and (c) wave-1 component of the geopotential height field, (d) mean zonal flow ( $\left.\mathrm{mm} \mathrm{s}^{-1}\right),(\mathrm{e})$ wave-2 and (f) wave-4 component of radial velocity amplitude. 